\title{
Numerical Modeling of Flows of Different Plasma Constituting Gases in RF-Plasmatron Facility
}

\author{
V.I. Vlasov, G.N. Zalogin, R.V. Kovalev \\ FGUP «TSNIImash», Korolev, Moscow Region, 141070, Russia \\ zalogin_gn@mail.ru
}

\begin{abstract}
Numerical results of plasma flow parameter studies a represented in the paper for a high temperature facility with inductively coupled $1 \mathrm{MWt}$ power plasmatorch (RF-plasmatron) operating with different work gases. Argon, nitrogen, air and carbon dioxide/nitrogen mixture (corresponding to Martian atmosphere) were chosen for the study. Modelling the problem of discharge chamber flow was based on mutual consideration of Navier-Stokes and Maxwell equations at given mass flow rates $(G=5 \div 30 \mathrm{~g} / \mathrm{s})$ over pressure range of $p=10 \div 200$ mbar and delivered power (energy supply) of $N=50 \div 300 \mathrm{kWt}$. Values of parameters at the discharge chamber exit (Fig. 1) were used then for computation of flow in the work chamber and around test models and probes (Fig. 2). Gas mixture models accounting for nonequilibrium thermochemical processes were utilized under solution of the problems. Developed soft ware is used for both plasma flow diagnostics and interpretation of tests on heat transfer, specification of thermal stability and catalicity of heat shield materials.
\end{abstract}

Keywords: RF-plasmatron, discharge chamber, nonequilibrium gas flow.

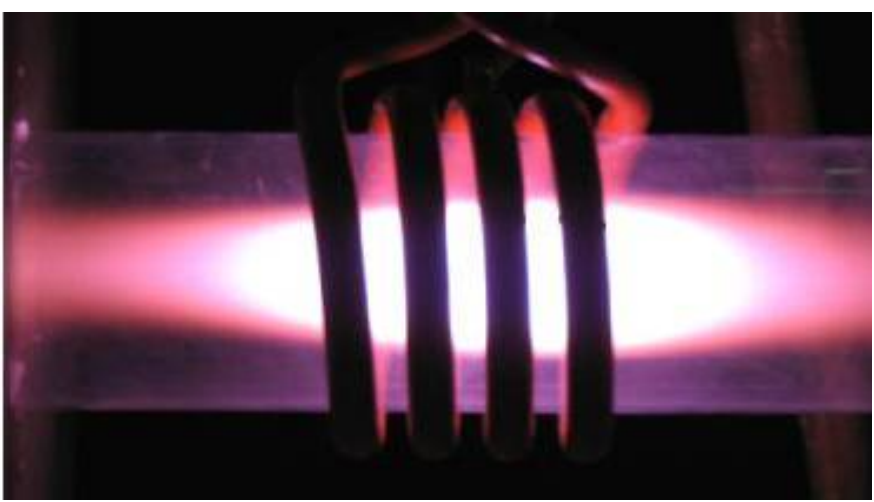

Fig. 1. Photo of RF-plasmatron discharge chamber flow

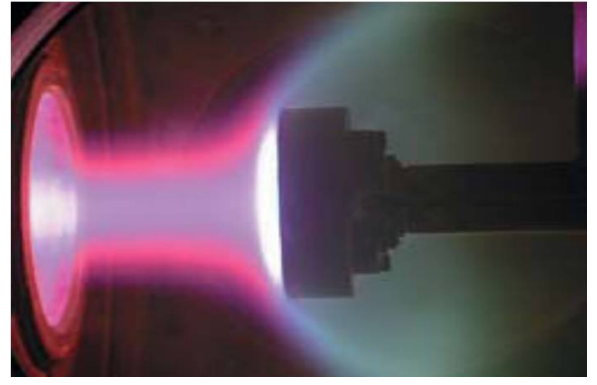

Fig. 2. Flow around a test model in plasma jet 


\title{
Численное моделирование течения различных плазмообразующих газов в тракте ВЧ плазмотрона
}

\author{
В.И. Власов, Г.Н. Залогин, Р.В. Ковалёв \\ Центральный научно-исследовательский институт машиностроения \\ Россия, Моск. обл., г. Королев, 141070, ул. Пионерская, 4 \\ zalogin_gn@mail.ru
}

\begin{abstract}
Аннотация
В работе представлены результаты численных исследований параметров плазменных потоков в высокотемпературной установке с высокочастотным индукционным плазмотроном (ВЧ плазмотроном) мощностью 1 МВт при работе на различных плазмообразующих газах. В качестве таких газов были выбраны: аргон, азот, воздух смесь углекислого газа с азотом (смесь, соответствующая атмосфере Марса). Решение задачи о течении газа в разрядной камере ВЧ плазмотрона основано на совместном рассмотрении уравнений НавьеСтокса и Максвелла при заданных расходах газа $G=5 \div 30$ г/с, в диапазоне давлений $p=10 \div 200$ ГПа, и потребляемой мощности (энерговклада) $N=50 \div 300$ кВт. Значения параметров на выходе из разрядной камеры (рис. 1) используются для расчета течения газа в рабочей камере установки и обтекании моделей и зондов (рис. 2). При решении задач используются модели газовых смесей, учитывающие неравновесное протекание физико-химических процессов. Разработанный программный комплекс используется при проведении диагностики плазменных струй и при интерпретации результатов экспериментов по теплообмену, определению термостойкости и каталитичности материалов тепловой защиты.
\end{abstract}

Ключевые слова: ВЧ плазмотрон, разрядная камера, неравновесный газовый поток.

\section{1. Введение}

В настоящее время всё более широкое применение получают установки с безэлектродным высокочастотным (ВЧ) нагревом газа. Приоритет в данном направлении исследований принадлежит России [1-4 и др.]. Позднее установки с ВЧ нагревом газа были созданы во Франции, Бельгии, Японии, США, Китае. Эти установки имеют целый ряд преимуществ перед высокотемпературными установками других типов, в том числе:

- $\quad$ отсутствие загрязняющих примесей в потоке газа;

- $\quad$ возможности воспроизведения и изменения определяющих параметров потока (полная энтальпия, давление, состав газа) в широком диапазоне для моделирования реальных условий на наиболее теплонапряженном участке полета ЛА;

- $\quad$ возможности испытаний крупномасштабных образцов ТЗМ и фрагментов конструкций, по длительности соответствующих реальному спуску в атмосфере $(t \sim 1200$ с для многоразовых ЛА).

К числу решаемых на установках с ВЧ подогревом газа задач, в частности, относятся:

- у уточнение теплообмена в высокотемпературных потоках химически неравновесных газов;

- $\quad$ исследование термостойкости материалов при их обтекании диссоциированным воздухом или углекислым газом; 
- $\quad$ отработка противоокислительных покрытий для теплозащитных материалов многоразового использования;

- $\quad$ ресурсные испытания элементов теплозащитных систем;

- $\quad$ определение каталитических свойств материалов по отношению к реакциям рекомбинации атомов;

- $\quad$ исследование совместимости материалов в плазменном потоке в зоне их контакта;

- $\quad$ исследование прохождения и затухания радиоволн в плазме, а также влияние на эти процессы продуктов разрушения ТЗМ;

- $\quad$ исследование механизмов возбуждения электронных состояний молекул и атомов и излучения.

Совокупность расчетных и экспериментальных данных, получаемых в ВЧ плазмотронах, могут быть использованы также для валидации расчетных и физико-химических моделей неравновесных газовых смесей.

Отработка тепловой защиты гиперзвуковых летательных аппаратов (ЛА) является весьма сложной комплексной научно-технической проблемой, требующей разработки методов и средств регистрации и определения многих параметров (в том числе, теплофизических и абляционных характеристик, термостойкости и каталитической активности исследуемых материалов). Применение стандартных методов исследований, разработанных для диапазона умеренных температур, часто становится затруднительным, а порой и невозможным в экстремальных условиях обтекания моделей и образцов ТЗМ высокоэнтальпийными потоками диссоциированного и частично ионизованного газа. Постоянное совершенствование как экспериментальных установок, используемых при отработке ТЗМ, так и средств измерений сопровождается возрастанием требований к точности определяемых параметров.

При проведении исследований в потоках низкотемпературной плазмы как связанных с их диагностикой, так и в описанных выше практических приложениях, количество параметров, подлежащих прямым измерениям, крайне ограничено. Поэтому важным научным направлением, расширяющим границы проводимых исследований, является численное моделирование течений высокотемпературных смесей газов, нагреваемых ВЧ разрядом, а также решения задач обтекания и теплообмена моделей и зондов с учетом многочисленных физико-химических процессов, протекающих неравновесным образом.

Принцип действия таких установок основан на известном физическом явлении нагрева проводящих сред электрическим током, индуцированным в них переменным электромагнитным полем. В высокочастотных плазмотронах подобной нагреваемой проводящей средой является ионизованный газ. Переменное электромагнитное поле создается внутри индуктора (это катушка-многовитковый (обычно 3-5 витков) соленоид). В индуктор помещается цилиндрическая разрядная камера из радиопрозрачного материала (как правило, кварцевого стекла), через которую протекает рабочий газ.

Детальное описание течения в ВЧ плазмотроне можно получить путем совместного решения уравнений газодинамики с учетом реальных свойств газа и уравнений Максвелла.

\section{2. Постановка задачи}

Проблеме численного моделирования течения высокотемпературной плазмы в разрядной камере ВЧ плазмотрона к настоящему времени посвящено немало публикаций. В ранних работах задача рассматривалась преимущественно в равновесной постановке [5-7]. Также были проведены расчёты неравновесного разряда в аргоне [8, 9]. С начала 2000-х годов стали появляться расчёты неравновесной воздушной плазмы в однотемпературной и многотемпературной постановках. Не претендуя на полноту, укажем лишь на некоторые работы [10-15].

Применительно к установке ВГУ-4 ИПМех РАН для неравновесного воздуха рассматриваемая задача решена в [16]. Для установки ВАТ-104 с ВЧ плазмотроном ЦАГИ решение задачи численного моделирования течения описано в $[17,18]$. 
Ниже рассматриваются вопросы комплексного моделирования течения в тракте установки с ВЧ плазмотроном $[7,11]$. Элементами тракта, подлежащими рассмотрению, являются: разрядная камера, сопловой блок, свободная струя, выходящая из сопла и область вокруг моделей или зондов, обтекаемых плазменной струёй.

В число определяемых параметров входят: составляющие скорости газа, поступательная, колебательная и электронная температуры плазмы, концентрации всех учитываемых компонентов, включая электроны. Кроме того, в разрядной камере и вокруг неё определяются характеристики высокочастотного электромагнитного поля.

Разработанный программный комплекс широко используется как при предварительном анализе возможностей моделирования условий теплообмена образцов ТЗМ и элементов конструкций, так и при интерпретации экспериментальных данных по диагностике параметров струи и определении наиболее важных характеристик, таких как термостойкость и каталитичность.

\section{1. Газодинамическая система уравнений}

Систему уравнений для дозвукового стационарного осесимметричного течения смеси газов в разрядной камере индукционного ВЧ плазмотрона в цилиндрической системе координат $(x, y)$ можно записать в следующем виде:

Уравнение неразрывности для смеси в ичелом

$$
\frac{\partial \rho u y}{\partial x}+\frac{\partial(\rho v y)}{\partial y}=0
$$

Уравнения движения

$$
\begin{gathered}
\frac{\partial}{\partial x}\left[y\left(\rho u^{2}+p-\tau_{x x}\right)\right]+\frac{\partial}{\partial y}\left[y\left(\rho u v-\tau_{x y}\right)\right]=y F_{x} \\
\frac{\partial}{\partial x}\left[y\left(\rho u v-\tau_{x y}\right)\right]+\frac{\partial}{\partial y}\left[y\left(\rho v^{2}+p-\tau_{y y}\right)\right]=p+\rho w^{2}-\tau_{\varphi \varphi}+y F_{y}, \\
\frac{\partial}{\partial x}\left[y\left(\rho u w-\tau_{x \varphi}\right)\right]+\frac{\partial}{\partial y}\left[y\left(\rho v w-\tau_{y \varphi}\right)\right]=-\rho v w+\tau_{y \varphi}
\end{gathered}
$$

где $\mathbf{U}=(u, v, w)$ - осевая, радиальная и тангенциальная компоненты вектора скорости; $\mathbf{F}$ - вектор магнитной силы Лоренца; $\mu, p, \rho-$ вязкость, давление и плотность газовой смеси; а вязкие потоки равны

$$
\begin{aligned}
& \tau_{x x}=\mu\left(2 \frac{\partial u}{\partial y}-\frac{2}{3} \operatorname{div} \mathbf{U}\right), \tau_{y y}=\mu\left(2 \frac{\partial v}{\partial y}-\frac{2}{3} \operatorname{div} \mathbf{U}\right), \tau_{x y}=\mu\left(\frac{\partial u}{\partial x}+\frac{\partial v}{\partial y}\right), \\
& \tau_{\varphi \varphi}=\mu\left(2 \frac{v}{y}-\frac{2}{3} \operatorname{div} \mathbf{U}\right), \tau_{x \varphi}=\mu \frac{\partial w}{\partial y}, \tau_{y \varphi}=\mu\left(\frac{\partial w}{\partial y}-\frac{w}{y}\right), \quad \operatorname{div} \mathbf{U}=\frac{\partial u}{\partial x}+\frac{1}{y} \frac{\partial v y}{\partial y}
\end{aligned}
$$

Уравнения диффузии химических компонентов

$$
\frac{\partial}{\partial x}\left[y\left(\rho u c_{i}-J_{i x}\right)\right]+\frac{\partial}{\partial y}\left[y\left(\rho v c_{i}-J_{i y}\right)\right]=y \dot{\omega}_{i},
$$

где $\mathbf{J}_{i}$-диффузионный поток и $\dot{\omega}_{i}$-скорость образования $i$-го компонента за счёт химических реакций 


$$
\dot{\omega}_{i}=\sum_{s}\left(v_{i s}^{\prime \prime}-v_{i s}^{\prime}\right)\left[K_{f s} \prod_{j}\left(\frac{\rho_{j}}{M_{j}}\right)^{v_{j s}^{\prime}}-K_{r s} \prod_{j}\left(\frac{\rho_{j}}{M_{j}}\right)^{v_{j s}^{\prime \prime}}\right]
$$

В выражении для источников (2.4) $K_{f s}, K_{r s}-$ константы скоростей прямых и обратных реакций; $v_{i s}^{\prime}, v_{i s}^{\prime \prime}$ - стехиометрические коэффициенты; $c_{i}, \rho_{i}=\rho c_{i} ; M_{i}$-массовая концентрация, плотность и молекулярный вес $i$-го компонента.

Уравнение сохранения полной энергии, записанное для полной энтальпии $H_{0}$

$$
\frac{\partial}{\partial x}\left[y\left(\rho u H_{0}-q_{x}\right)\right]+\frac{\partial}{\partial y}\left[y\left(\rho v H_{0}-q_{y}\right)\right]=y \dot{Q}_{J}+\Phi^{*}
$$

Здесь $\dot{Q}_{J}-$ джоулево тепло; $H_{0}=\sum_{i} c_{i} h_{i}+0.5 U^{2}-$ полная энтальпия газа; $h_{i}-$ энтальпия $i$-го компонента. Вязкая диссипация $\Phi^{*}$ равна

$$
\Phi^{*}=\frac{\partial}{\partial x}\left[y\left(u \tau_{x x}+v \tau_{x y}+w \tau_{x \varphi}\right)\right]+\frac{\partial}{\partial y}\left[y\left(u \tau_{x y}+v \tau_{y y}+w \tau_{y \varphi}\right)\right]
$$

Тепловой поток в газовой смеси за счёт теплопроводности и диффузии $\mathbf{q}$ определяется выражением

$$
\mathbf{q}=-\lambda_{t r} \nabla T-\sum_{m} \lambda_{v m} \nabla T_{\mathrm{vm}}-\lambda_{e} \nabla T_{e}+\sum_{i} h_{i} \mathbf{J}_{i}
$$

Уравнения для колебательных энергий

$$
\frac{\partial}{\partial x}\left[y\left(\rho u e_{v m}-q_{v x}\right)\right]+\frac{\partial}{\partial y}\left[y\left(\rho v e_{v m}-q_{v y}\right)\right]=y \dot{Q}_{m},
$$

где $e_{v m}$ - колебательная энергия молекул сорта $m ; \mathbf{q}_{v}=\left(q_{v x}, q_{v y}\right)$ - тепловой поток этой энергетической моды за счёт градиента колебательной температуры $T_{v m}$; и диффузии молекул

$$
\mathbf{q}_{v}=-\lambda_{v m} \nabla T_{v m}+\mathbf{J}_{m} e_{e m}
$$

$\dot{Q}_{m}=\dot{Q}_{m}^{V T}+\dot{Q}_{m}^{V V}+\dot{Q}_{m}^{C T}-$ скорость обмена колебательной энергии молекул сорта $m$ вследствие колебательно-поступательного обмена, колебательно-колебательного обмена (с другими молекулами) и за счёт химических реакций (включая процессы возбуждения-тушения электронных состояний).

Уравнение баланса энергии электронного газа (для электронной температуры $\left.T_{e}\right)$

$$
\frac{\partial}{\partial x}\left[y\left(n_{e} u \frac{5}{2} k T_{e}-q_{e x}\right)\right]+\frac{\partial}{\partial y}\left[y\left(n_{e} u \frac{5}{2} k T_{e}-q_{e y}\right)\right]=y\left(\dot{Q}_{e-T}+\dot{Q}_{e-V}+\dot{Q}_{I}+\dot{Q}_{J}\right)
$$

Здесь $\dot{Q}_{e-T}-$ скорость энергообмена между электронами и поступательной модой;

$\dot{Q}_{e-V}-$ между электронами и колебательной энергией;

$\dot{Q}_{I}-$ скорость потерь энергии свободных электронов на ионизацию.

Тепловой поток за счёт электронной теплопроводности и диффузии электронов равен [5]

$$
\mathbf{q}_{e}=-\lambda_{e} \nabla T_{e}+\frac{5}{2} k T_{e} \mathbf{J}_{e}
$$




\section{Электромагнитная система уравнений}

Для численного моделирования электромагнитного поля в плазменного разряда будем использовать осесимметричные уравнения Максвелла и простейшую форму закона Ома [19]

$$
\nabla \times \mathbf{B}=\frac{4 \pi}{c} \mathbf{j}, \quad \nabla \times \mathbf{E}=-\frac{1}{c} \frac{\partial \mathbf{B}}{\partial t}, \quad \mathbf{j}=\chi \mathbf{E}
$$

В (2.11) В и $\mathbf{E}$ - магнитное и электрическое поля; $\mathbf{j}$ - плотность электрического тока; $\chi$ - проводимость плазмы. Токами смещения по сравнению с токами проводимости пренебрегаем, магнитная проницаемость равна 1. Также необходимо отметить, что для типичных частот ВЧ генераторов ( $f=0.44 \div 1.76$ МГц) проводимость не зависит от частоты поля [19].

В осесимметричном приближении электромагнитное поле реального индуктора заменяется полем $N_{c}$ круговых витков радиуса $a$, по которым течет ток $I_{0} \exp (-i \omega t)$ с круговой частотой $\omega=2 \pi f[20]$

$$
\begin{gathered}
E_{0 \varphi}(x, y)=\frac{4 i \omega}{c^{2}} I_{0} \sqrt{\frac{a}{y} \sum_{l=1}^{N c}\left[\left(1-\frac{k_{l}^{2}}{2}\right) K^{*}\left(k_{l}\right)-E^{*}\left(k_{l}\right)\right],} \\
k_{l}^{2}=\frac{4 a y}{(a+y)^{2}+\left(x-x_{l}\right)^{2}},
\end{gathered}
$$

где $x_{l}$ - координаты витка с номером $l ; K^{*}(k)$ и $E^{*}(k)$ - полные эллиптические интегралы первого и второго рода. В такой постановке уравнения Максвелла сохраняют свою линейную структуру (коэффициенты не зависят от поля), и при гармонической внешней нагрузке поле плазмы будет также меняться по времени по гармоническому закону. Удобно использовать комплексные амплитуды электрического $\widehat{\mathbf{E}}=\mathbf{E}_{1}+i \mathbf{E}_{2}$ и магнитного полей $\widehat{\mathbf{B}}=\mathbf{B}_{1}+i \mathbf{B}_{2}$, так что

$$
\mathbf{E}=\widehat{\mathbf{E}} \exp (-i \omega t), \quad \mathbf{B}=\widehat{\mathbf{B}} \exp (-i \omega t)
$$

Уравнения (2.11) при этих предположениях можно переписать в следующем виде:

$$
\nabla \times \widehat{\mathbf{B}}=\frac{4 \pi}{c} \chi \widehat{\mathbf{E}}, \quad \nabla \times \widehat{\mathbf{E}}=\frac{i \omega}{c} \widehat{\mathbf{B}}
$$

Исключая $\widehat{\mathbf{B}}$, имеем уравнение для амплитуды $\widehat{\mathbf{E}}$

$$
\nabla \times \nabla \times \widehat{\mathbf{E}}=\frac{4 \pi i \omega}{c^{2}} \widehat{\mathbf{E}}
$$

В осесимметричном случае от нуля отличны только тангенциальная компонента $\widehat{E}_{\varphi}$ комплексной амплитуды вектора $\widehat{\mathbf{E}}$, а также осевая $\widehat{B}_{x}$ и радиальная $\widehat{B}_{y}$ компоненты вектора $\widehat{\mathbf{B}}$. Переходя к координатному представлению, $(2.13, \sigma)$ можно записать в следующем, удобном для численного интегрирования виде:

$$
\frac{\partial^{2} \widehat{E}_{\varphi}}{\partial x^{2}}+\frac{\partial}{\partial y}\left(\frac{1}{y} \frac{\partial y \widehat{E}_{\varphi}}{\partial y}\right)=-\frac{4 \pi i \omega \chi}{c^{2}} \widehat{E}_{\varphi}
$$

Пространство, в котором необходимо определить электромагнитное поле, не совпадает с областью газодинамического течения плазмы, т.к. оно существует и вне разрядной камеры. Поэтому здесь используется подход, позволяющий найти решение электромагнитной задачи во всём пространстве $[7,10,12]$. На основе принципа линейной суперпозиции амплитуду $\widehat{E}_{\varphi}$ 
можно представить в виде суммы поля индуктора $\widehat{E}_{0 \varphi}(2.12)$ и поля $\widehat{e}$, индуцированного токами в плазме

$$
\widehat{E}_{\varphi}=\widehat{E}_{0 \varphi}+\hat{e}
$$

Переходя от комплексных амплитуд к действительной и мнимой частям

$$
\hat{e}=e_{1}+i e_{2}, \quad \bar{E}_{0 \varphi}=i E_{0},
$$

из комплексного уравнения (2.14) получаем систему двух действительных уравнений для определения величин $e_{1}$ и $e_{2}$

$$
\begin{gathered}
\frac{\partial^{2} e_{1}}{\partial x^{2}}+\frac{\partial}{\partial y}\left[\frac{1}{y} \frac{\partial\left(y e_{1}\right)}{\partial y}\right]-4 \pi \chi \frac{\omega}{c^{2}} e_{2}=4 \pi \chi \frac{\omega}{c^{2}} E_{0}, \\
\frac{\partial^{2} e_{2}}{\partial x^{2}}+\frac{\partial}{\partial y}\left(\frac{1}{y} \frac{\partial\left(y e_{2}\right)}{\partial y}\right)+4 \pi \chi \frac{\omega}{c^{2}} e_{1}=0
\end{gathered}
$$

При известном электрическом поле магнитное поле определяется с помощью соотношений $(2.13, a)$

$$
\mathbf{B}_{1}=\frac{c}{\omega} \nabla \times \mathbf{E}_{2}=\frac{c}{\omega}\left(\begin{array}{l}
\frac{1}{y} \frac{\partial y\left(E_{0}+e_{2}\right)}{\partial y} \\
-\frac{\partial\left(E_{0}+e_{2}\right)}{\partial x}
\end{array}\right), \quad \mathbf{B}_{2}=-\frac{c}{\omega} \nabla \times \mathbf{E}_{1}=-\frac{c}{\omega}\left(\begin{array}{l}
\frac{1}{y} \frac{\partial y e_{1}}{\partial y} \\
-\frac{\partial e_{1}}{\partial x}
\end{array}\right)
$$

Усредняя по времени выражение для силы Лоренца, получим выражение для магнитной силы в уравнениях движения (2.2)

$$
\mathbf{F}=\frac{1}{c} \overline{\mathbf{j} \times \mathbf{B}}=\frac{\chi}{2 \omega}\left(\mathbf{E}_{1} \times \mathbf{B}_{1}+\mathbf{E}_{2} \times \mathbf{B}_{2}\right)
$$

Выражение для расчёта джоулева тепла получается аналогично путём усреднения мгновенной скорости диссипации электромагнитной энергии

$$
\dot{Q}_{J}=\overline{\mathbf{j} E}=\frac{\chi}{2}\left[e_{1}^{2}+\left(e_{2}+E_{0}\right)^{2}\right]
$$

\section{2. Постановка сопряжённой задачи}

При дозвуковом истечении плазмы ВЧ разряда в рабочую часть установки поставленная задача является эллиптической. В этом случае задача расчета течений в разрядной камере и рабочей частях должна решаться совместно. Разработанные численные методы и программы позволяют анализировать течения газа как в отдельных областях (разрядная камера и рабочая часть с обтекаемой моделью) без учёта влияния их друг на друга, так и на основе совместного решения задачи во всей объединённой области. Как показывают расчеты, в большинстве случаев обратным влиянием течения газа в рабочей части на течение в разрядной камере можно пренебречь. Некоторое влияние наблюдается, когда модель больших размеров установлена близко к выходному сечению сопла.

Рассмотрим постановку задачи о течении плазмы в разрядной камере ВЧ плазмотрона. Подача рабочего газа обычно осуществляется через набор отверстий, расположенных в форме кольца. В осесимметричной постановке они заменяются концентрической щелью такого же радиуса и площади. Суммарный расход и температура рабочего газа задаются (измеряются экспериментально). Таким образом, при заданной геометрии, включая угол закрутки потока, 
можно определить все компоненты вектора скорости на входной границе. В итоге на входе в разрядную трубу $(x=0)$ имеем

$$
u=u_{\infty}, v=0, w=w_{\infty}, T=T_{\infty}, c_{i}=c_{i \infty}, T_{v m}=T_{e}=T_{\infty}
$$

На оси симметрии $(y=0)$ ставятся обычные условия

$$
v=w=0, \quad \frac{\partial u}{\partial y}=\frac{\partial T}{\partial y}=\frac{\partial c_{i}}{\partial y}=\frac{\partial T_{v m}}{\partial y}=\frac{\partial T_{e}}{\partial y}=0
$$

На дозвуковой выходной границе ставятся следующие условия: давление $p$ задано, а для всех остальных переменных - линейная экстраполяция на границе, т.е.

$$
p=p_{\text {out }}, \frac{\partial^{2} f}{\partial x^{2}}=0, \text { где } f=u, v, w, T, c_{i}, T_{v m}, T_{e}
$$

При численном моделировании условий экспериментов берётся измеренное манометром в рабочей камере значение $p_{\text {out }}$.

На твёрдых границах разрядной камеры можно ограничиться упрощёнными граничными условиями (эффектами скольжения, гетерогенной рекомбинации, аккомодации колебательной энергии пренебрегается) - условия прилипания для скорости и поступательной температуры и нулевые градиенты для концентраций и колебательно-электронных температур

$$
u=v=w=0, T=T_{w}(x), \quad \frac{\partial c_{i}}{\partial y}=\frac{\partial T_{v m}}{\partial y}=\frac{\partial T_{e}}{\partial y}=0
$$

Здесь $T_{w}(x)$ - переменная температура твёрдых поверхностей разрядной камеры. На поверхности кварцевой трубки $T_{w} \sim 350 \div 700 \mathrm{~K}$, а на охлаждаемых металлических стенках $T_{w} \sim 300 \mathrm{~K}$.

Численные расчёты подтверждают, что на параметры в ядре плазменной струи учёт более точных условий на стенках разрядной камеры почти не влияет.

Уравнения Максвелла (2.15) решаются в такой области пространства (вообще говоря, не совпадающей с областью газодинамического течения), где электромагнитное поле существенно. Внешнюю границу полезно совместить со стенкой металлического кожуха, окружающего разрядную камеру или расположить там, где электрическое поле практически равно нулю (свободная граница). Такой подход позволяет учесть влияние внешних металлических частей плазмотрона на поле внутри разрядной камеры.

На металлической стенке имеем следующее условие (поле в металл не проникает):

$$
e_{1}=e_{2}+E_{0}=0
$$

На оси симметрии

$$
e_{1}=e_{2}=0
$$

На свободной границе с нормалью $n$ используется линейная экстраполяция

$$
\frac{\partial^{2} e_{1}}{\partial n^{2}}=\frac{\partial^{2} e_{2}}{\partial n^{2}}=0
$$

Корректная постановка электромагнитной задачи должна включать себя расчёт цепи ВЧ генератора для определения амплитуды тока в индукторе $I_{0}(2.12)$. Дело в том, что электрическое поле плазменных токов $\hat{e}$ создаёт в индукторе значительную ЭДС, и величина $I_{0}$ не может быть определена до решения всей задачи. Чтобы обойти эту трудность используется следующий приём - при численном моделировании задаётся не ток $I_{0}$, а мощность, вложенная в плазму 


$$
N_{p l}=2 \pi \iint Q_{J}(x, y) y \mathrm{~d} y \mathrm{~d} x
$$

Так как решение уравнений Максвелла (2.15) пропорционально току в индукторе, величина $I_{0}$ корректируется в процессе вычислений, чтобы удовлетворить условию (2.26).

\section{3. Результаты расчётов течения в ВЧ плазмотроне при работе с различными плазмообразующими газами}

Расчеты проводились для ВЧ плазмотрона ЦНИИмаш (установка У-13 ВЧП) мощностью $N=1 \mathrm{MB}$ (длина разрядной камеры с сопловым блоком $L=0.85 \mathrm{м}$, внешний радиус $R=0.1 \mathrm{м}$, частота генератора $f=440$ кГц) для чистых газов и их смесей, используемых в задачах наземной экспериментальной отработки изделий РКТ. К таким газам относятся: воздух (смесь азота и кислорода), марсианская атмосфера (смесь углекислого газа $\mathrm{CO}_{2}$, азота и аргона), чистый азот, чистый аргон. Все расчеты проводились для наиболее общего случая неравновесного протекания физико-химических процессов.

\section{Аргон}

Проведения испытаний ТЗМ в потоке аргона иногда необходимы, чтобы исключить влияние различных гетерогенных процессов на поверхности материала или реакций диссоциированного воздуха с газообразными продуктами абляции, а также при нанесении покрытий, для предотвращения окисления используемого материала. В частично ионизованном аргоне при расчетах учитывается три компонента: $\mathrm{Ar} \mathrm{Ar}^{+}$и электроны.

Расчёты проводились для режима работы ВЧ плазмотрона при расходе плазмообразующего газа $G=5$ г/с, давлении $p=50$ гПа и энерговкладе по анодному питанию $N=200$ кВт. Отметим, что экспериментальным исследованиям ВЧ разряда в аргоновой плазме при атмосферном давлении уделялось значительное внимание в 60-70 гг. прошлого века. Этот вопрос подробно изложен в [4].

На рис. 1-3 показаны поля параметров в разрядной камере ВЧ плазмотрона. На рис. 1 в верхней части рисунка показаны изолинии функции тока, а на нижней - поле энтальпии газа. Как следует из представленных данных даже при достаточно высокой вкладываемой мощности $N=200$ кВт, максимальное значение энтальпии $H_{0}$ не превышает 3.5 МДж/кг, что существенно (почти в 10 раз) меньше, чем при тех же условиях в потоках двух (азот, воздух) и трехатомных $\left(\mathrm{CO}_{2}\right)$ газов. Это связано с тем, что в аргоне ВЧ пробой и горение разряда происходит при малой степени ионизации $\left(\alpha=5 \times 10^{-3}\right)$, когда затрачиваемая на этот процесс энергия мала. При этом реализуемая энтальпия $H_{0}=c_{p} T$. Теплоёмкость аргона при $T \sim(6 \div 10) \times 10^{3} \mathrm{~K}$, $c_{p} \approx 0.5$ КДж/(кг К). Измерения КПД установки при работе на аргоне, проведенные в [21], также показали малые (10\%) значения этого параметра.

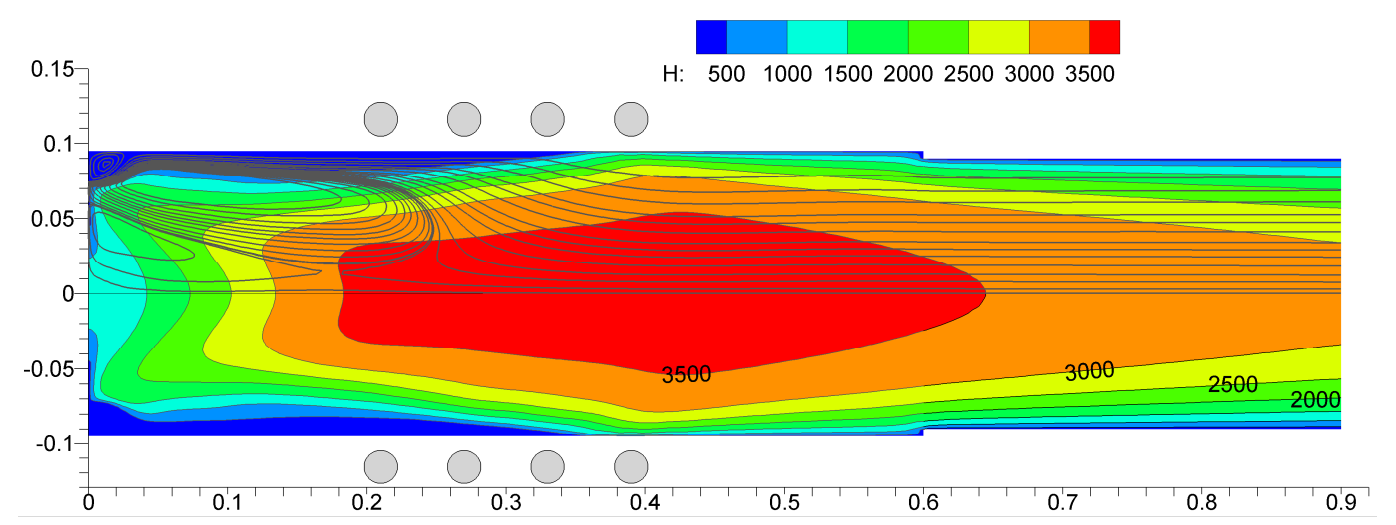

Рис. 1. Изолинии функции тока (верхняя часть) и поле энтальпии (нижняя часть) в разрядной камере, $p=50$ гПа, $N=200$ кВт 
На рис. 2 показаны поля электронной (сверху) и поступательной температур (снизу) в разрядной камере.

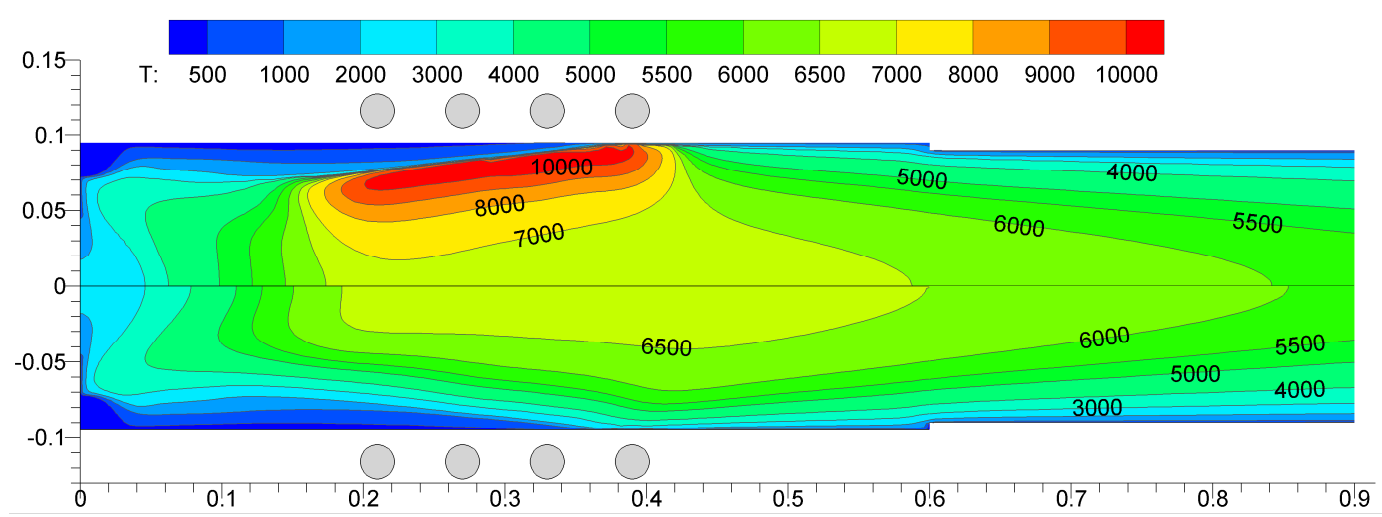

Рис. 2. Поля электронной (сверху) и поступательной температур (снизу) в разрядной камере, $p=50$ гПа, $N=200$ кВт

Электронная температура в области энерговклада превышает поступательную. Это связано с тем, что энергия электромагнитного поля первоначально поглощается электронами, которые передают её тяжелым частицам, находящимся в смеси. Из рисунка видно, что область энерговклада (скин-слой) с повышенной температурой электронов при работе ВЧ плазмотрона на аргоне, смещена к стенке разрядной камеры. При выходе газа в рабочую камеру установки электронная температура практически сравнивается с поступательной.

На рис. 3 показано поле объёмной плотности энергии $W\left(\mathrm{BT} / \mathrm{cm}^{3}\right)$, поглощаемой плазмой в разрядной камере. Здесь же приведены силовые линии (линии постоянной напряженности в В/см) электрического поля.

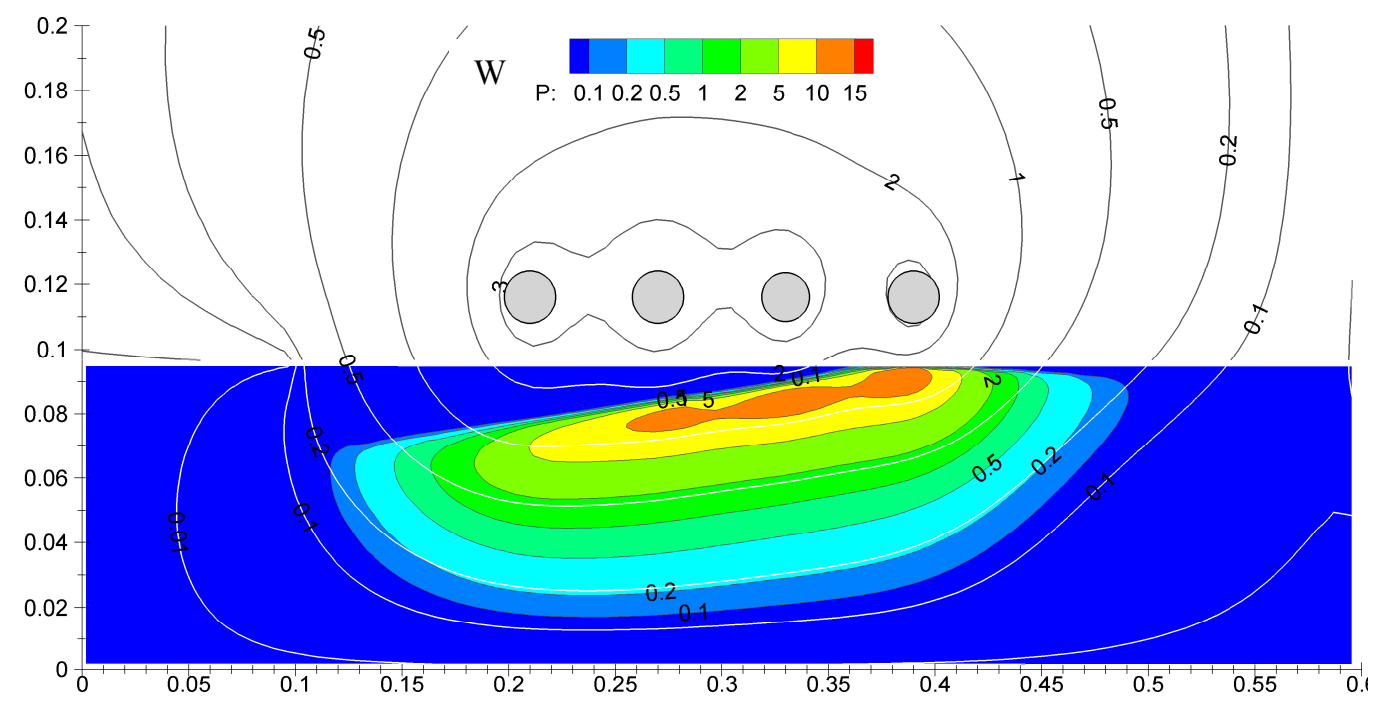

Рис. 3. Поле объёмной плотности энергии, поглощаемой плазмой в разрядной камере, и силовые линии электрического поля

Данные, приведенные на этом рисунке по распределению величины объёмной плотности энергии коррелируют с повышенным значением температуры электронов вблизи стенки разрядной камеры, показаном на рис. 2. Однако при пониженных давлениях (в данном случае $p=50$ гПа) локальные максимумы температуры быстро исчезают за счет влияния диффузии и теплопроводности. При этом на выходе из разрядной камеры не наблюдается максимума температуры, который характерен при $p=10^{5}$ Па [4]. 
На рис. 4 представлено поле электронных концентраций $n_{e}$ в разрядной камере. В области разряда (2-4 витки идуктора, см. рис. 3) из-за смещения зоны энерговклада с повышенной температурой к стенке вблизи неё реализуются высокие значения $n_{e}$. Всю область электроны заполняют за счёт процесса амбиполярной диффузии. В центральной части струи эти значения сохраняются на достаточно большом расстоянии от зоны энерговклада из-за достаточно медленно процесса трехчастичной рекомбинации.

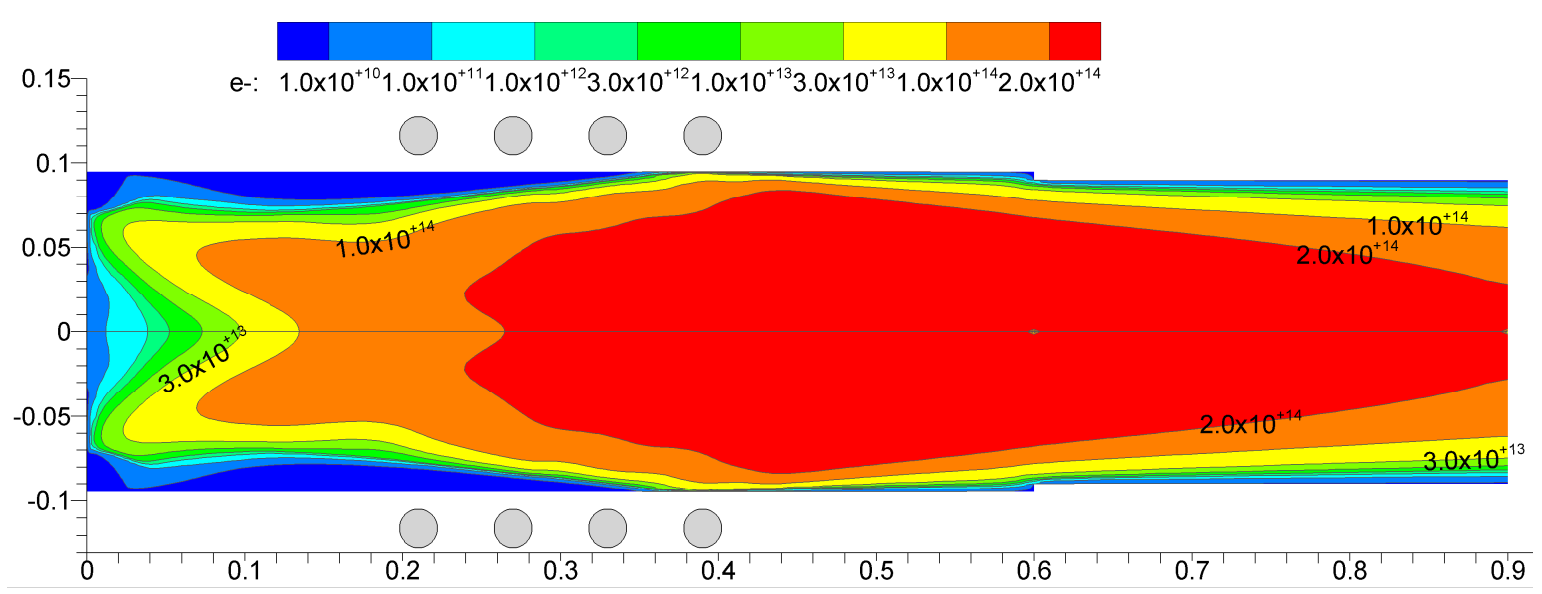

Рис. 4. Электронная концентрация $n_{e}\left(1 / \mathrm{cm}^{3}\right)$ в разрядной камере, $p=50$ гПа, $N=200$ кВт

Быстрая релаксация температур в рассматриваемом случае может быть связана с существенно меньшими градиентами параметров при частоте генератора $f=440$ кГц. Плазмотроны меньших размеров, описанные в [4], как правило, работают на существенно больших частотах $f \sim 5 \div 30$ МГц. При этом толщина скин-слоя $\Delta \sim \sqrt{1 / \chi f}$, где $\chi-$ проводимость плазмы, зависящая от рода газа, его температуры и давления, а $f$ - частота электромагнитного поля, существенно меньше (а градиенты величин существенно больше), чем в большом плазмотроне.

\section{Азот}

Проведение экспериментальных исследований взаимодействия диссоциированного азота с материалами тепловой защиты как многоразовыми, так и аблирующими, необходимо для нахождения вероятностей (констант скоростей) реакций, описывающих такие процессы. При исследованиях влияния на теплообмен многоразовых материалов их каталитических свойств в отношении реакций рекомбинации атомов и построению моделей таких процессов, первоначально должны быть определены вероятности реакций в чистых газах. В тракт плазмотрона азот подается из баллона.

Аналогичная задача возникает и при диагностике высокоэнтальпийных диссоциированных газов (в частности, определение энтальпии газа $H_{0}$ ) с помощью калориметрических датчиков теплового потока. Измеряемая величина теплового потока в диссоциированном газе в значительной степени зависит от каталитичности поверхности тепловоспринимающего элемента (ТВЭ), который обычно выполняется из металла (меди, серебра и др.). Погрешность измерения теплового потока, связанная с неточностью используемого при обработке данных значения каталитичности датчика, непосредственно переносится и на получаемую величину $H_{0}$. В кислородосодержащих газах (воздухе или $\mathrm{CO}_{2}$ ) возникает дополнительная сложность при интерпретации результатов измерений теплового потока, связанная с окислением ТВЭ и изменением его каталитических свойств. В диссоциированном азоте эта проблема исключается.

При расчетах течения диссоциированного и частично ионизованного азота учитывается пять компонентов: $\mathrm{N}_{2}, \mathrm{~N}, \mathrm{~N}_{2}^{+}, \mathrm{N}^{+}$и электроны. Важным отличием молекулярных газов от одноатомных является быстрый процесс передачи энергии электронов в энергию колебаний мо- 
лекул. Этот процесс протекает с образованием отрицательного молекулярного иона $\mathrm{N}_{2}^{-}$, который быстро распадается на колебательно-возбуждённую молекулу и электрон с меньшей энергией [19]. Этот процесс препятствует значительному превышению температуры электронов над колебательной температурой молекул в области разряда.

В качестве примера расчета течения в разрядной камере и сопловом блоке (сопло с выходным сечением $D=124$ мм) для условий экспериментов в азоте при давлении $p=12$ гПа, расходе $G=5$ г/с и энерговкладе по анодному питанию $N=300$ кВт на рис. $5, a-2$ показаны поля полной энтальпии газа $H_{0}$ и изолинии функции тока $(a)$, поступательной температуры $T$ (б), электронно-колебательная температура (b), а также поля концентраций атомов азота $C_{\mathrm{N}}(2)$.

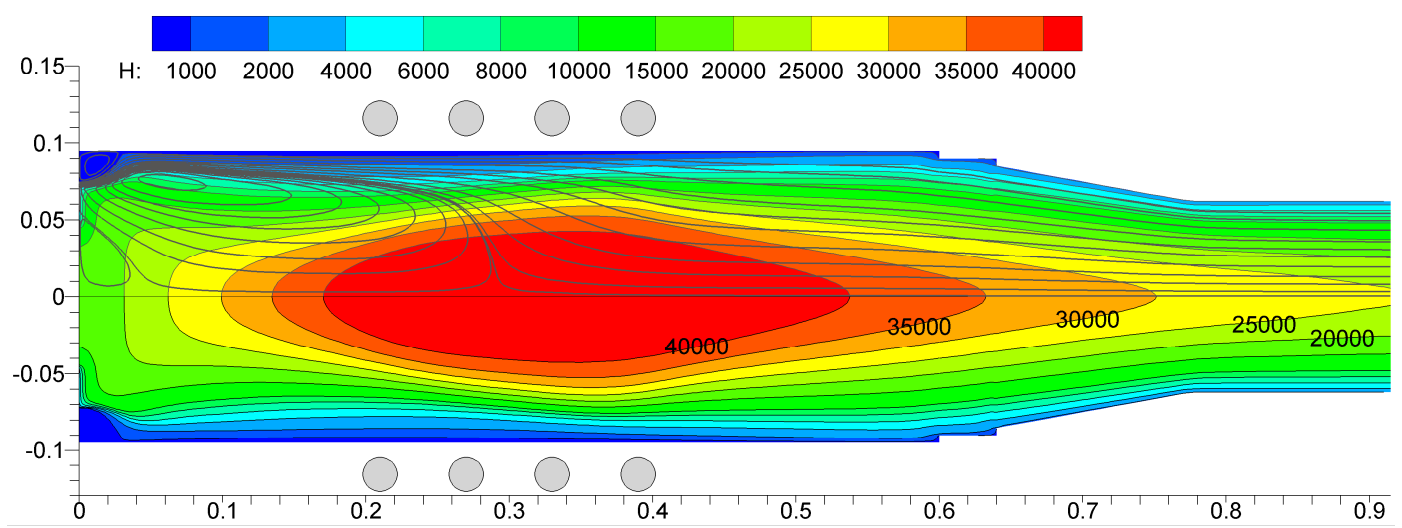

Рис. $5, a$. Изолинии функции тока тока и поле энтальпии в разрядной камере, $p=12$ гПа, $N=300$ кВт

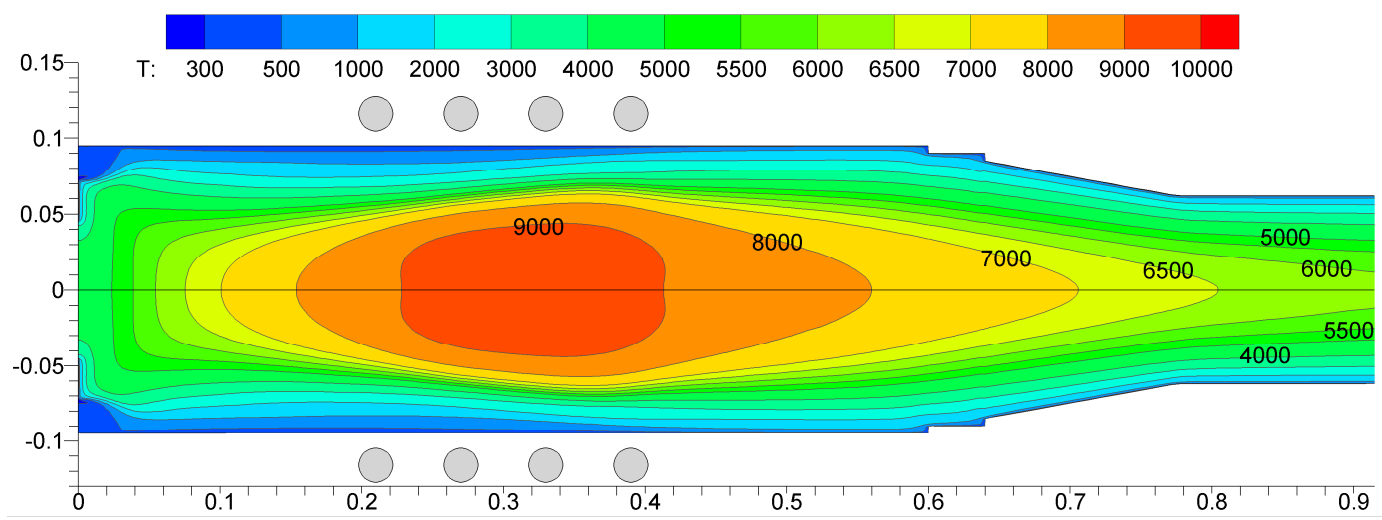

Рис. 5, б. Поле поступательной температуры в разрядной камере, $p=12$ гПа, $N=300$ кВт

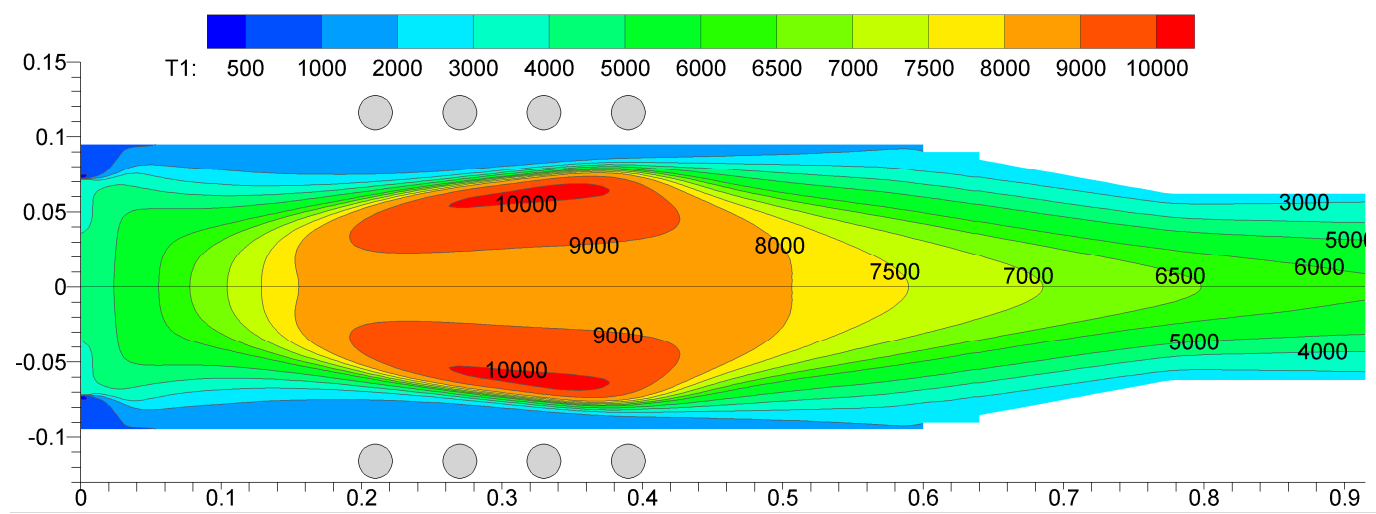

Рис. 5, в. Электронно-колебательная температура в разрядной камере, $p=12$ гПа, $N=300$ кВт 


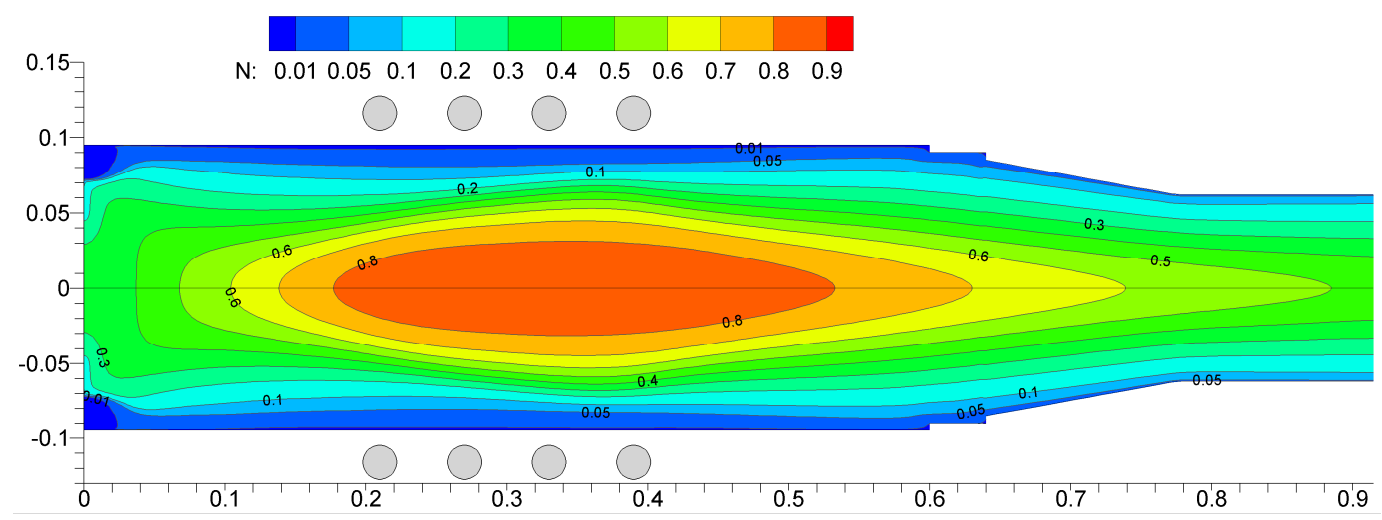

Рис. 5, г. Массовая концентрация атомов азота $C_{\mathrm{N}}$ в разрядной камере, $p=12$ гПа, $N=300$ кВт

Значение кпд установки при расчётах азота и воздуха брались по данным прямых измерений [21]. Эта величина зависит от давления газа $p$ и энерговклада $N$ (по анодному питанию). При малых энерговкладах и высоком давлении кпд достигают 50 \%, уменьшаясь по мере возрастания величины $N$. Для азота в расчётах при $N=300$ кВт и $p=12$ гПа принято значение кпд $\eta=0.25$, а для воздуха при $N=100$ кВт, $p=25$ гПа кпд $\eta=0.35$.

В области индуктора электронно-колебательная температура также, как и температура электронов в случае аргона, превышает поступательную. Однако это превышение не столь значительно. На выходе из соплового блока температуры выравниваются и приобретают профиль, близкий к параболическому.

Как показывает сравнение результатов расчетов течения в разрядной камере, выполненных с использованием равновесной и неравновесной моделями газов, на выходе из разрядной камеры (или соплового блока) параметры газа (температуры и концентрации компонентов) на оси струи при $p \leq 25$ гПа отличаются от равновесных. С увеличение давление это различие уменьшается. Поэтому при анализе теплообмена моделей в дозвуковом потоке в рамках неравновесного пограничного слоя на его внешней границе вместо энтальпии задаётся температура и состав газа, полученные из расчёта обтекания на основе уравнений Навье-Стокса.

Отметим, что в молекулярных газах и их смесяхв зоне разряда образование «затравочных» электронов для лавинной ионизации атомов электронным ударом, обусловлено быстрыми реакциями с образованием молекулярных ионов (в азоте $\mathrm{N}_{2}^{+}$, а в воздухе $\mathrm{NO}^{+}$). В аргоне образование «затравочных» электронов связано с медленным процессом возбуждения электронных состояний с их последующей ионизацией [19]. Это приводит к существенному различию полей температуры в разрядной камере в аргоне и молекулярных газах.

Как следует из представленных данных, в зоне разряда реализуются следующие максимальные значения параметров: $H_{0} \approx 40$ МДж/кг, $T \approx 9000 \mathrm{~K}, T_{e v} \approx 10000 \mathrm{~K}, C_{\mathrm{N}} \approx 0.8$. На выходе из соплового блока в рабочую камеру установки эти же параметры принимают значения (на оси струи) $H_{0} \approx 25 \mathrm{MДж/кг,} \mathrm{поступательная} \mathrm{температура} \mathrm{газа} T \approx 6000 \mathrm{~K}$, электронноколебательная температура $T_{e v} \approx 7000 \mathrm{~K}$, массовая концентрация атомов $C_{\mathrm{N}} \approx 0.45$.

Картина течения и поля параметров около модели в виде цилиндрического торца диаметром 70 мм показаны рис. $6, a-6$. На рис. $6, a$ приведено поле энтальпий, на рис. 6,6 - поле температуры (выше оси симметрии) и концентрации атомов азота (нижняя часть). Проведенные для данного режима расчеты пограничного слоя на критической линии тока около модели, свидетельствуют о замороженности химических реакций.

\section{Воздух}

Наибольшее количество экспериментов по отработке ТЗМ и определению их свойств проводится в воздухе. В 11-компонентной физико-химической модели воздуха $\left(\mathrm{O}_{2}, \mathrm{~N}_{2}, \mathrm{NO}, \mathrm{O}\right.$, $\mathrm{N}, e^{-}, \mathrm{NO}^{+}, \mathrm{N}_{2}^{+}, \mathrm{O}_{2}^{+}, \mathrm{N}^{+}, \mathrm{O}^{+}$) учитываются диссоциация, ионизация, обменные и ионно-молекулярные реакции, протекающие неравновесным образом. 


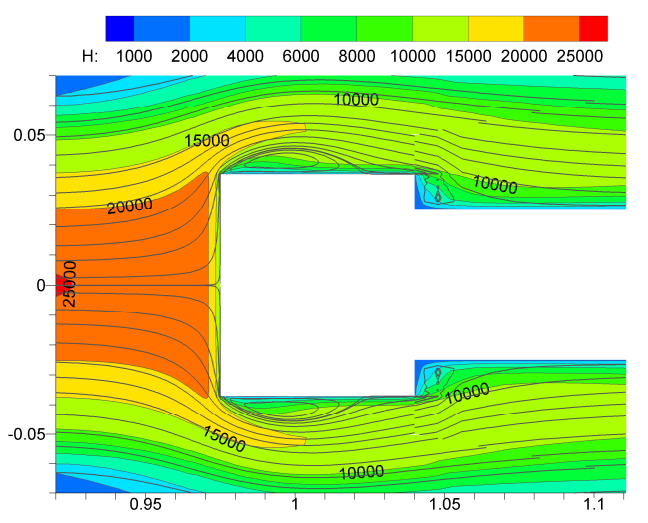

Рис. 6, a. Линии тока и поле энтальпии вокруг поверхности державки

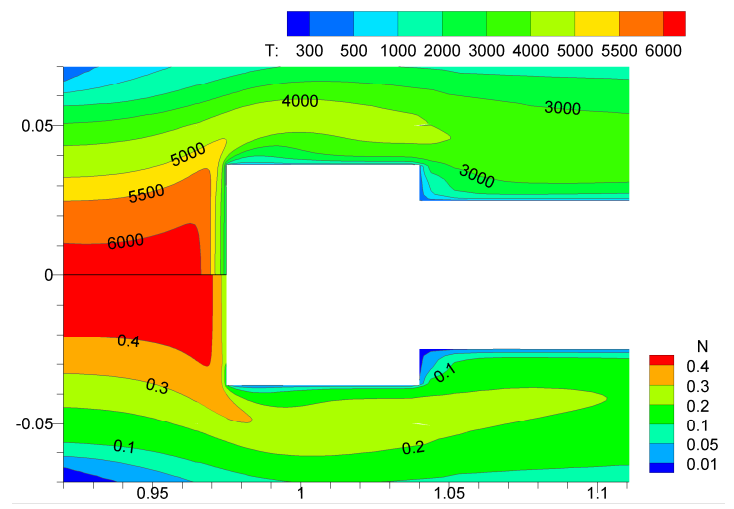

Рис. 6, б. Поле температур (верх) и массовая концентрация атомов азота (снизу)

Модель газа, включая расчёт коэффициентов переноса и гетерогенных реакций, подробно описана в [22].

Для расчета коэффициентов диффузии смеси используется самосогласованный метод эффективных коэффициентов бинарной диффузии (SCEBD) (Ramshaw J.D., 1990).

При этом диффузионный поток $J_{i}$ представляется в виде двух слагаемых - градиентного члена (закон Фика) и корректирующей поправки $\alpha_{i}$

$$
\mathbf{J}_{i}=-\rho D_{i} \nabla c_{i}+\boldsymbol{\alpha}_{i}
$$

Выражение для эффективного коэффициента диффузии $D_{i}$ можно получить из соотношений Стефана-Максвелла

$$
D_{i}=\frac{1-x_{i}}{\sum_{j \neq i} x_{j} / D_{i j}}
$$

Коэффициенты бинарной диффузии $D_{i j}$ рассчитываются через интегралы столкновений. С учётом обязательного условия $\sum_{i} \mathbf{J}_{i}=0$ поправку $\alpha_{i}$ можно приближённо выразить в форме $\boldsymbol{\alpha}_{i}=\rho c_{i} \sum_{j} D_{j} \nabla c_{j}$ и записать выражение для диффузионного потока в виде

$$
\mathbf{J}_{i}=-\rho D_{i} \nabla c_{i}+\rho c_{i} \sum_{j} D_{j} \nabla c_{j}
$$

Для расчётов коэффициентов диффузии заряженных частиц используется модель амбиполярной диффузии [19].

Гетерогенные процессы на стенках разрядной камеры, сопле и в рабочей части учитываются приближенно (формулы (2.19-2.22)). На экспериментальных моделях и образцах исследуемых материалов возможно два способа учёта конечной каталитичности поверхности. В первом случае, который используется в представленных для иллюстрации расчётах, задаётся вероятность рекомбинации атомов $\gamma$ или связанная с ней константа скорости каталитической рекомбинации $K_{w}$. В этом случае граничные условия для концентраций атомов и молекул записываются в виде

$$
J_{i}=K_{w i} \rho_{w} C_{i w}, \quad i=\mathrm{O}, \mathrm{N} ; \quad J_{m}=-J_{a}
$$

Поскольку процесс гетерогенной рекомбинации атомов многостадийный, то величина $K_{w}$ строго говоря является эффективной константой, характеризующей скорость протекания процесса в целом. 
Во втором случае учитываются отдельные стадии процесса рекомбинации, включающие процессы адсорбции-десорбции, рекомбинация атомов по механизмам Или-Райдила и Ленгмюра-Хиншельвуда. При этом вводится понятие степени заполнения поверхности частицами различного сорта $\theta_{i}$ [23]. Значения $\theta_{i}$ находятся из решения на каждой итерации алгебраической системы уравнений. При таком подходе исходными данными являются константы скоростей отдельных стадий, а значение $K_{w}$ определяется в процессе решения задачи. Поскольку упомянутые константы, как правило, не известны в практических приложениях такой подход используется редко.

Расчёты проводились при давлении и расходе воздуха $p=25$ гПа и $G=5$ г/с, энерговкладе по анодному питанию $N=100$ кВт. На рис. 7, $a-2$ приведены расчетные поля параметров в разрядной камере: поле полной энтальпии $H_{0}$ и изолинии функции тока; поле поступательной температуры $T$; поле электронно-колебательной температуры $T_{e-v i b}$; поле массовой концентрации атомов азота.

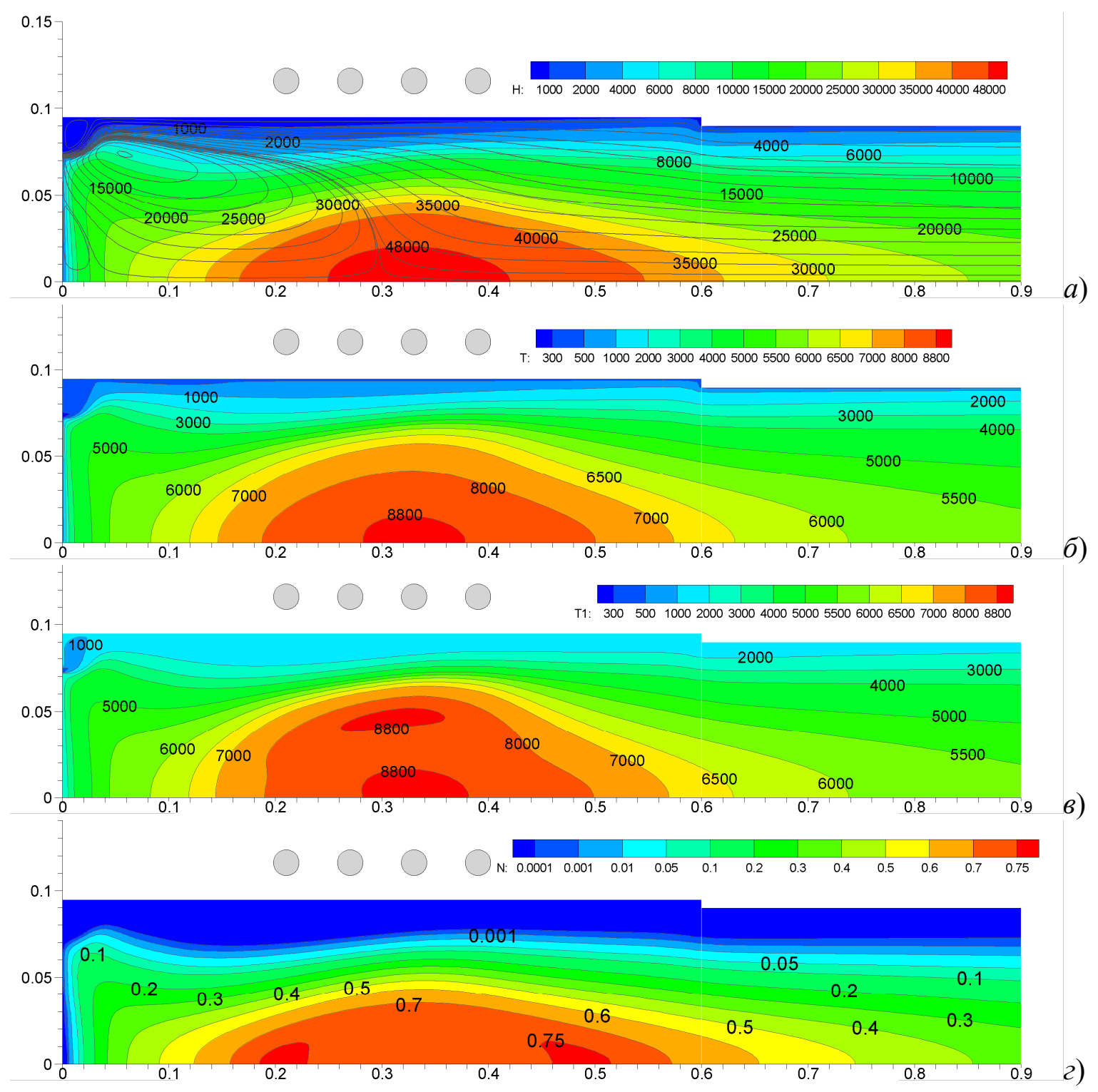

Рис. 7. Расчетные поля параметров в разрядной камере (воздушная плазма): $a$ ) поле полной энтальпии $H_{0}$ и изолинии функции тока; б) поле поступательной температуры $T$; в) поле электронно-колебательной температуры $T_{e-v i b} ;$ г) поле массовой концентрации атомов азота

Расчетные значения поступательной $T$ и электронно-колебательной температуры $T_{e-v i b}$ в центральной части струи близки. В зоне разряда энтальпия достигает значения $H_{0}=50$ МДж/кг, 
а массовая концентрация атомов азота $C_{\mathrm{N}} \approx 0.75$. То есть в потоке воздуха в зоне разряда газ (и кислород и азот) практически полностью диссоциирован.

На рис. 8, $a$ показаны расчетные распределения параметров $H_{0}, T, T_{e-v i b}$ на срезе соплового блока при работе ВЧ плазмотронана воздухе и азоте, а на рис. $8, \sigma$ - осевая скорость и окружная скорость. Как следует из представленных данных при одних и тех же условиях (по энерговкладу и давлению) энтальпия, температура и осевая скорость в воздухе несколько выше, чем в азоте. Окружные скорости близки. Электронно-колебательная температура в азоте $T_{e-v i b}$ при приближении к стенке остаётся существенно выше поступательной. Поскольку теплообмен модели в основном зависит от параметров струи в окрестности оси, то это различие в температурах не оказывает заметного влияния на тепловой поток.

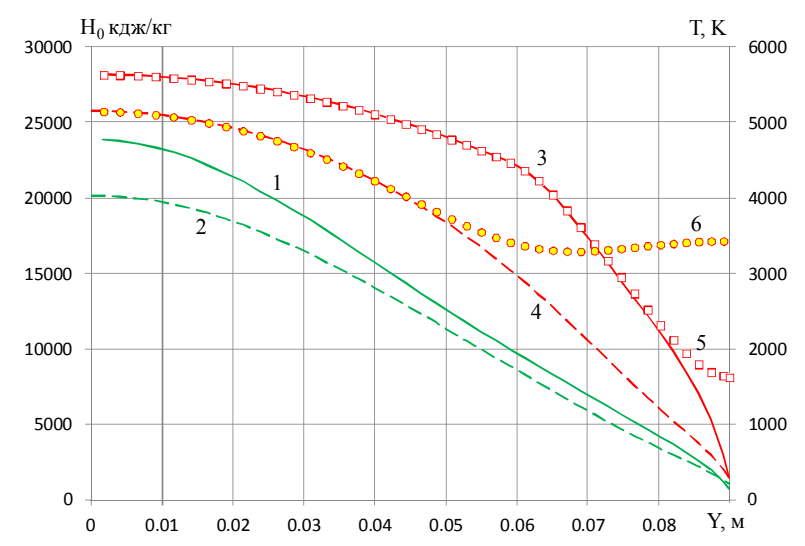

a)

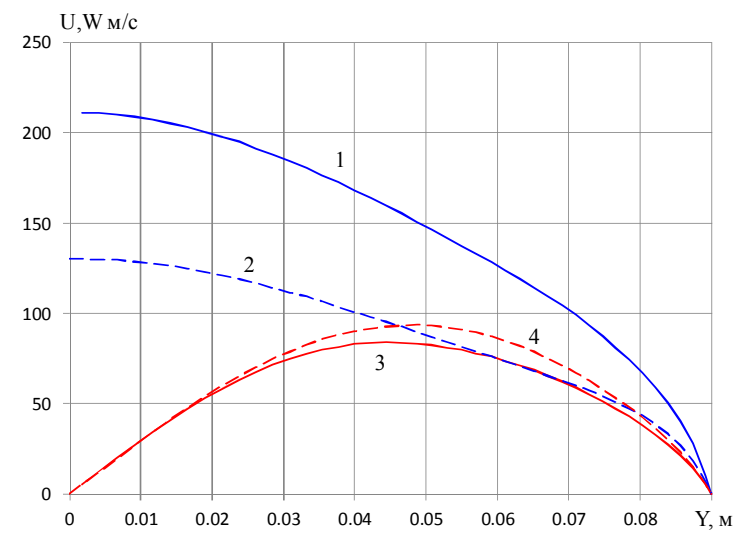

б)

Рис. 8. Распределение параметров плазменной струи на выходе из разрядной камеры: $a$ ) 1 - полная энтальпия $H_{0}$ (воздух), 2 - азот, 3 - поступательная температура $T$ (воздух), 4 - азот, 5 - электронноколебательная температура $T_{e-v i b}$ (воздух), 6 -азот; б) 1 - осевая скорость $U$ (воздух), 2 - азот, 3 окружная скорость $W$ (воздух), 4 - азот

На рис. 9, а показаны распределения полного давления $P_{0}=p_{c m}+\rho U^{2}$ и массового потока $\rho U$ в поперечном сечении струи воздуха и азота на выходе из разрядной камеры. На рис. 9, б приведены распределения массовых концентраций атомов азота и кислорода в выходном сечении разрядной камеры.

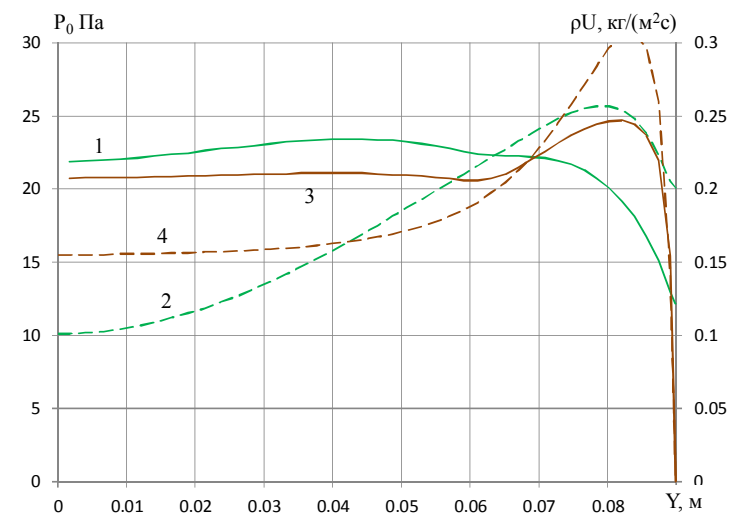

a)

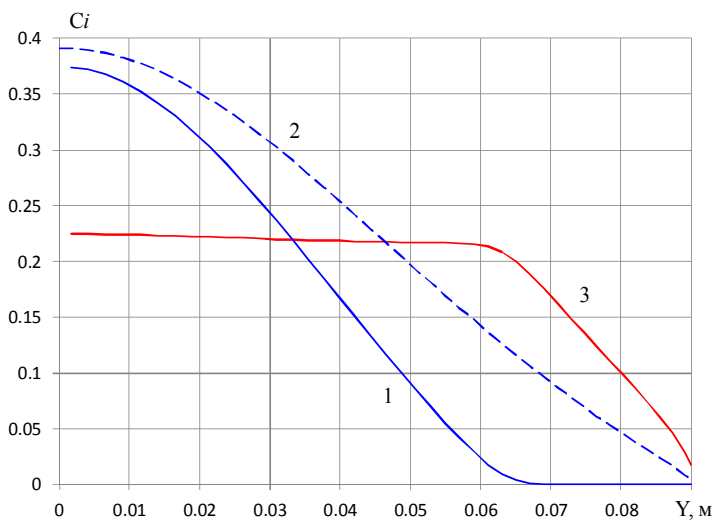

б)

Рис. 9. Распределение параметров плазменной струи на выходе из разрядной камеры: $a$ ) 1 - полное давление $P_{0}=p_{c m}+\rho U^{2}$ (воздух), 2 - азот, 3 - массовый поток $\rho U$ (воздух), 4 - азот; б) 1 - массовая концентрация атомов азота $C_{\mathrm{NB}}$ воздухе, 2 - в азоте, 3 - массовая концентрация атомов кислорода $C_{\mathrm{O}}$ в воздухе

Как следует из представленных данных, в потоке воздуха в большей части сечения струи кислород полностью диссоциирован (кривая 3). Распределение концентрации атомов азота по 
сечению струи для воздуха и азота существенно различаются, особенно на периферии струи. Это связано с тем, что в диссоциированном воздухе рекомбинация атомов азота протекает в основном в быстрых обменных реакциях с участием окиси азота NO, а в чистом азоте в медленно текущих реакциях трёхчастичной рекомбинации.

Полученные расчетные данные используются далее при расчетах обтекания и теплообмена моделей и зондов плазменной струёй.

На рис. 10 приведены расчетные распределения параметров в окрестности модели, состоящей из материалов с различной каталитической активностью, выполненной в виде цилиндрического торца диаметра $D=70$ мм, обтекаемой потоком воздуха [24]. Расчеты проводились на основе численного решения уравнений Навье-Стокса.

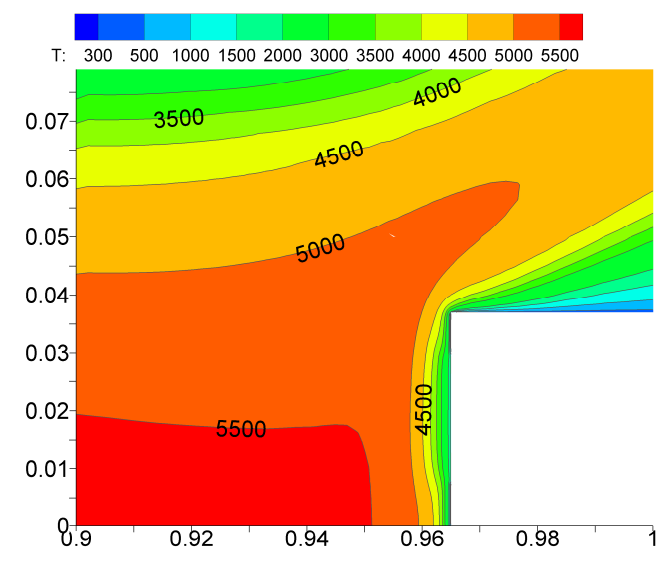

a)

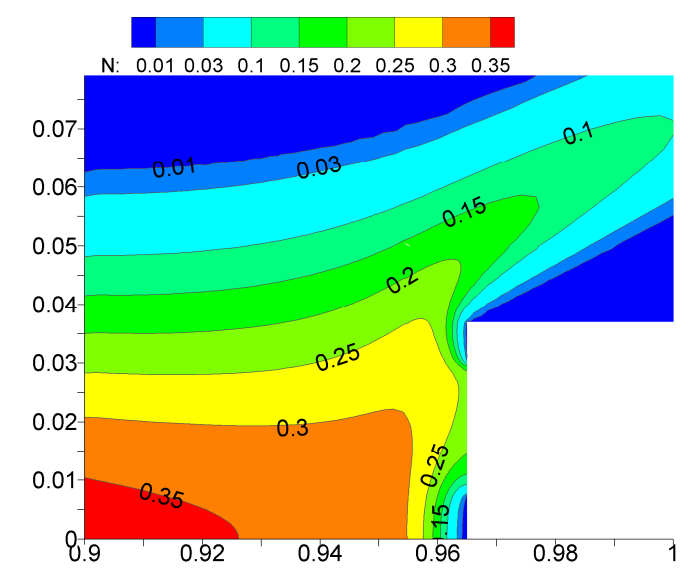

б)

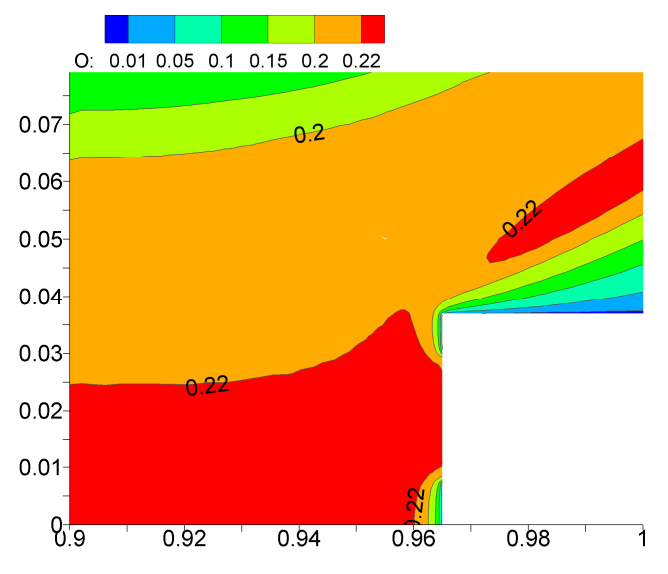

8)

Рис. 10. Распределение параметров в окрестности модели, обтекаемой струёй диссоциированного воздуха: $a$ ) температура газа; б) массовая концентрация атомов $\mathrm{N}$; ) массовая концентрация атомов $\mathrm{O}$

Режимы работы установки с повышенными значениями тепловых потоков реализуются при работе с соплами, имеющим выходное сечение $D=60$ и 30 мм. Режимы с максимальными значениями тепловых потоков реализуются в том случае, когда на выходе из сопла течение сверхзвуковое. Такие режимы показаны на рис. 11 и 12 для сопел с выходным сечением 60 и 30 мM.

В части струи, выходящей из сопла в рабочую часть, хорошо заметны бочки с повышенной температурой, характерные для сверхзвуковых струй.

Картина обтекания калориметрического зонда диаметром 50 мм сверхзвуковой струей при работе с соплом $D=30$ мм показана на рис. 13 . 


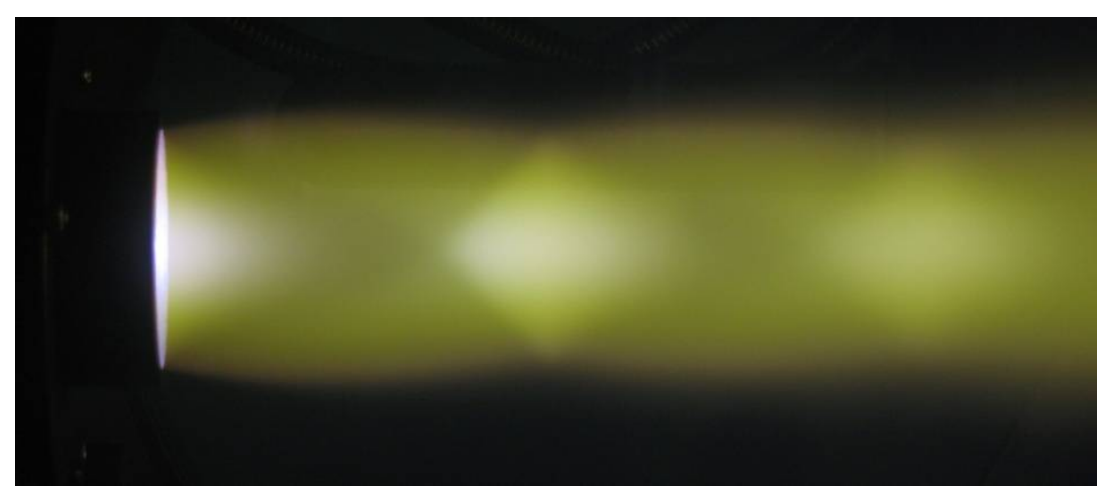

Рис. 11. Картина течения в свободной струе. Сопло $D=60$ мм, $N=200$ кВт

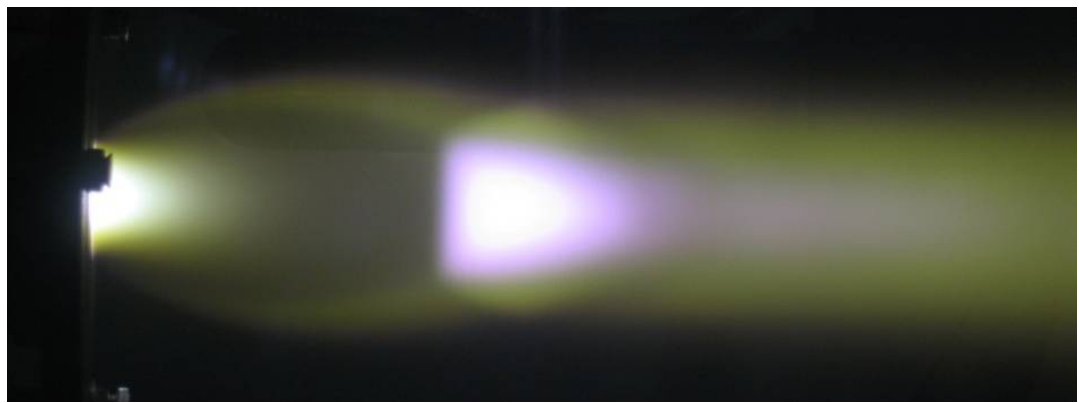

Рис. 12. Картина течения в свободной струе. Сопло $D=30$ мм, $N=300$ кВт

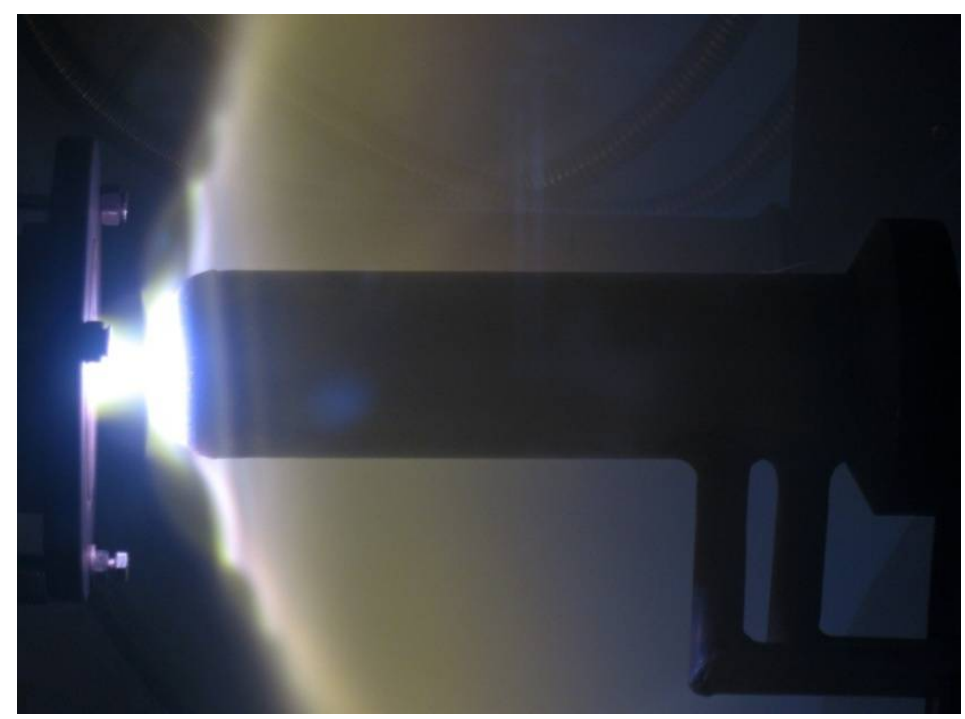

Рис. 13. Картина течения около калориметрического зонда. Сопло $D=30$ мм, $N=300$ кВт

Структура течения газа в недорасширенных струях, генерируемых в ВЧ плазмотроне, подробно рассмотрена в $[25,26]$.

На рис. 14 и 15 для $N=200$ кВт приведены профили параметров на срезе сопла $D=60$ мм. Величины температуры, скорости, давления, полной энтальпии и концентрации атомов азота плавно изменяются от своих максимальных значений на оси до стенки сопла. Концентрация атомов кислорода постоянна в бо́льшей части сечения сопла, что связано с полной диссоциацией молекул кислорода в рассматриваемых условиях. 


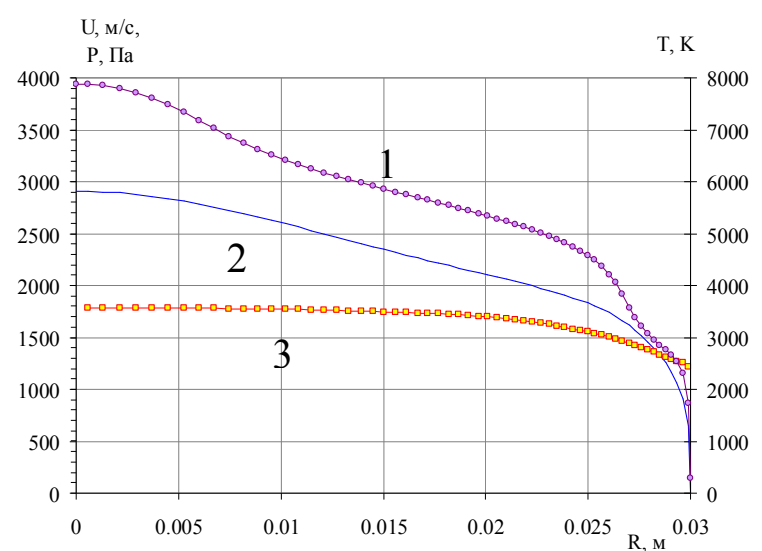

Рис. 14. Профили температуры (1), скорости (2) и давления (3) на срезе сопла. $N=200$ кВт

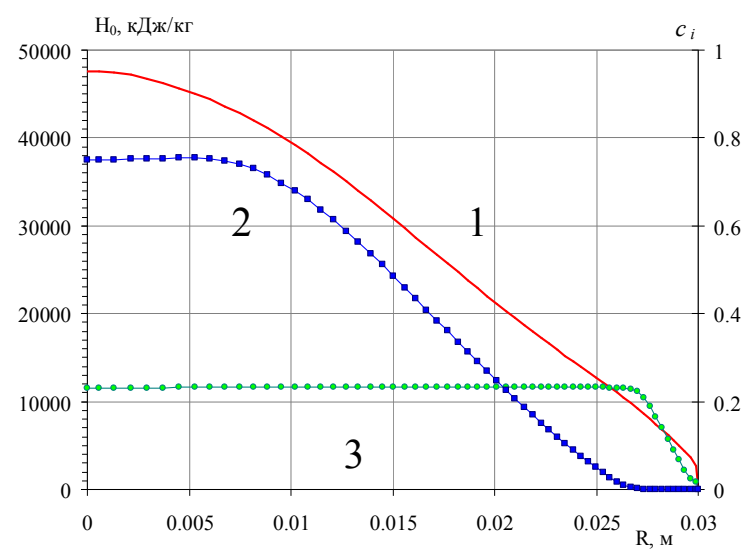

Рис. 15. Профили полной энтальпии $H_{0}(1)$, концентрации атомов азота $C_{\mathrm{N}}(2)$ и кислорода $C_{\mathrm{O}}$ (3) на срезе сопла. $N=200$ кВт

\section{Смесь углекислого газа $\left(\mathrm{CO}_{2}\right)$ с азотом $\left(\mathbf{N}_{2}\right)$}

Расчет течения такой смеси проводился с учетом следующих 15-ти компонентов: $\mathrm{CO}_{2}$, $\mathrm{CO}, \mathrm{O}_{2}, \mathrm{~N}_{2}, \mathrm{NO}, \mathrm{CN}, \mathrm{O}, \mathrm{N}, \mathrm{C}, e^{-}, \mathrm{CO}^{+}, \mathrm{NO}^{+}, \mathrm{C}^{+}, \mathrm{N}^{+}, \mathrm{O}^{+}$. Используемая физико-химическая модель таких смесей описана в [27]. Здесь показаны результаты расчетов, целью которых было моделирование условий теплообмена десантного модуля, соответствующих входу в атмосферу Марса со скоростью $V=5.2 \mathrm{kм} / \mathrm{c}$ (энтальпия торможения газа $H_{0}=13.5$ МДж/кг). При проведении экспериментальных исследований теплообмена заданные (достаточно низкие) значения энтальпии при относительно низких давлениях $p=10 \div 40$ гПа достигались установкой переходника в виде металлической трубы длиной $L=500$ мм между разрядной камерой и соплом за счёт тепловых потерь в металл.

В качестве примера результатов расчетов для таких смесей на рис. 16 показаны распределения линий тока и температуры газа в разрядной камере для варианта расчета: $p=40$ гПа, $N=135$ кВт, (КПД принимался равным 0.6). Для этих режимов на выходе из канала были получены близкие значения энтальпии $H_{0}=14.1$ МДж/кг. Температура газа на оси струи составляла $T \approx 3500 \mathrm{~K}$. Максимальное значение температуры в зоне разряда составляет $T \approx 7000 \mathrm{~K}$. Модель была расположена на оси потока на расстоянии $L=1.06$ м от среза кварцевой разрядной камеры.

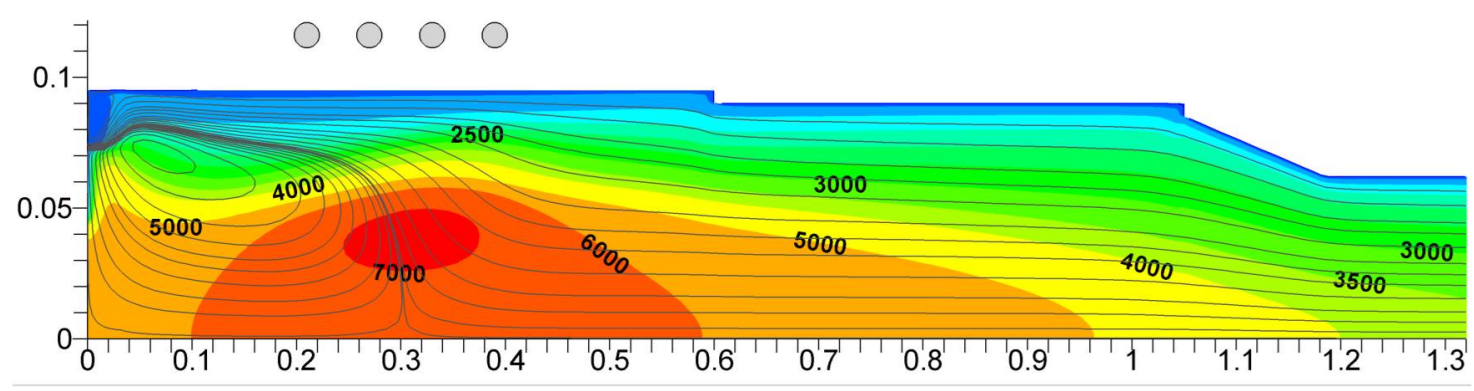

Рис. 16. Распределения изолиний функции тока и температуры газа в разрядной камере при $p=40$ гПа, $N=135$ кВт, энтальпия газа в выходном сечении сопла, $H_{0}=14.1$ МДж/кг

Результаты расчетов были использованы далее для определения параметров газа в струе и около зонда. На рис. 17 приведены линии тока и распределения температуры в струе и около зонда (цилиндр диаметром $D=50$ мм со скругленными кромками) для двух режимов работы ВЧ плазмотрона. 


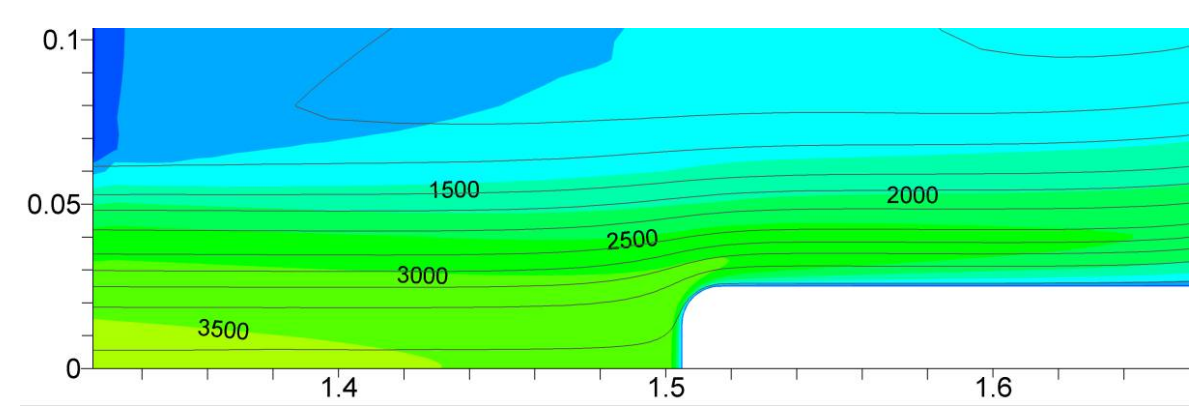

Рис. 17. Расчетные распределения температуры и изолинии функции тока около зонда при $p=40$ гПа, $N=135$ кВт, $H_{0}=14.1$ МДж/кг

На рис. 18 приведены расчетные распределения массовой концентрации атомов кислорода в струе и около зонда.

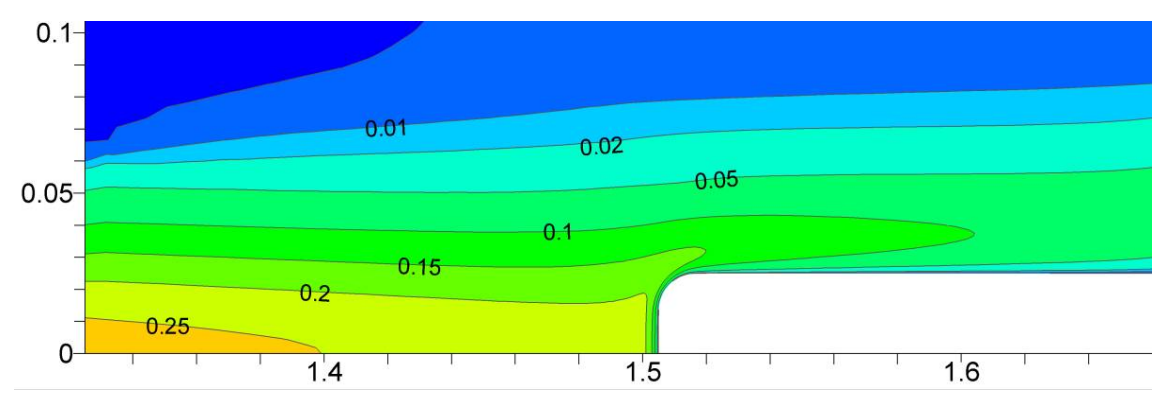

Рис. 18. Расчетное распределение массовых концентраций атомов О в условиях эксперимента при $p=40$ гПа, $N=135$ кВт, $H_{0}=14.1$ МДж/кг

Во всех расчетах на поверхности зонда для вероятности гетерогенной рекомбинации О и СО задавались значения $\gamma_{\mathrm{O}}=\gamma_{\mathrm{CO}}=0.01$, а вероятность адсорбции принималась равной $\gamma_{a d s}=0.2$. На внешней границе пограничного слоя при $p=10,40$ гПа задавалось значение температуры $T=3000 \div 3500 \mathrm{~K}$. Результаты этих расчётов подробно обсуждаются в [27].

Профили температуры, энтальпии и массовых концентраций основных компонентов на критической линии тока перед зондом для рассматриваемого случая приведены на рис. 19.

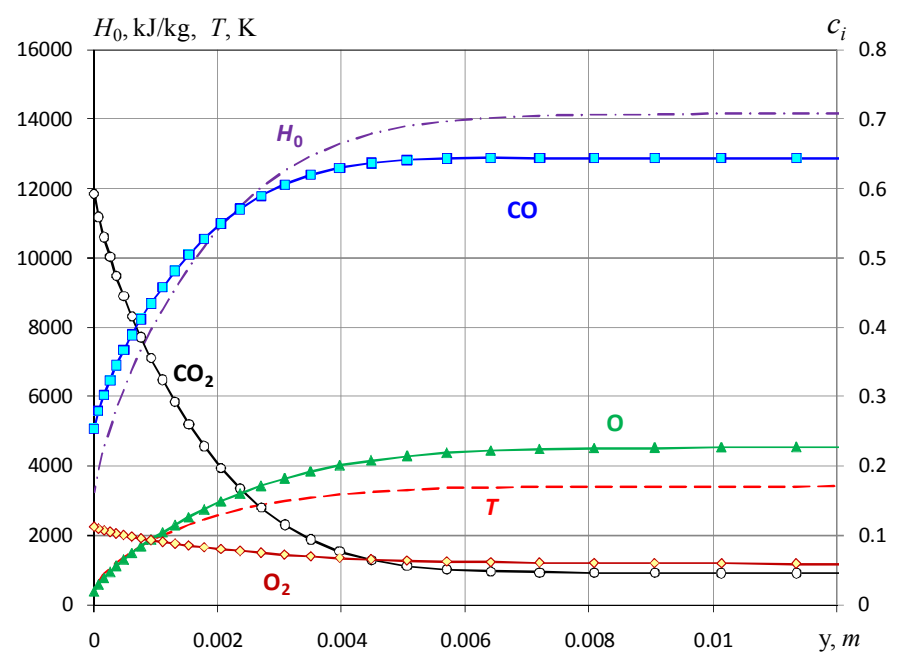

Рис. 19. Распределение энтальпии, температуры и массовых концентраций компонентов при $p=40$ гПа, $N=135$ кВт, $H_{0}=14.1$ МДж/кг 
Таким образом, разработанные численный метод и алгоритм решения задачи о течении различных газов в тракте установки с ВЧ плазмотроном, включающим четыре области: разрядную камеру, сопловой блок, струю в рабочей части и область около модели, позволяет получать распределение большого количества параметров. В число определяемых параметров входят: составляющие скорости газа, поступательная, колебательная и электронная температуры плазмы, концентрации всех учитываемых компонентов, включая электроны. Кроме того, в разрядной камере и вокруг неё определяются характеристики высокочастотного электромагнитного поля.

\section{4. Заключение}

На основе сравнения результатов расчётов неравновесного течения в тракте установки с ВЧ плазмотроном и около моделей, проведенных для различных плазмообразующих газов, а также с полученными ранее аналогичными расчётами с использованием равновесных физикохимических моделей можно сделать следующие выводы:

- $\quad$ на параметры газа в ядре плазменной струи, выходящей в рабочую камеру установки, учёт более точных условий на стенках разрядной камеры, таких как эффект скольжения, гетерогенной рекомбинации атомов, аккомодации колебательной энергии почти не влияет;

- $\quad$ в случае дозвукового течения задачи расчета течений в разрядной камере и рабочей части являются эллиптическими и должны решаться совместно. Разработанные численные методы и программы позволяют анализировать течения газа как в отдельных областях (разрядная камера и рабочая часть с обтекаемой моделью) без учёта влияния их друг на друга, так и на основе совместного решения задачи во всей объединённой области. Как показывают расчеты, в большинстве случаев обратным влиянием течения газа в рабочей части на течение в разрядной камере можно пренебречь. Некоторое влияние наблюдается, когда модель больших размеров установлена близко к выходному сечению сопла;

- $\quad$ неравновесные процессы, протекающие в разрядной камере, оказывают влияние на параметры плазменной струи на выходе из разрядной камеры в окрестности её оси при давлениях газа $p \leq 25$ гПа. На периферии струи некоторые параметры, например, электронно-колебательная температура газа может отличаться от поступательной и при больших давлениях. Этот эффект, однако, практически не влияет на величину теплового потока к модели. Значения параметров газа в струе, близкие к равновесным, позволяют проводить подробные исследования процессов в рамках пограничного слоя. Это существенно сокращает время расчёта и получения результата по сравнению с решением уравнений Навье-Стокса. В установках меньшей мощности, имеющих меньшие линейные размеры, это граничное значение давления может возрасти;

- $\quad$ при низких давлениях газа $p \leq 25$ гПа основные параметры (температура и состав газа) отличаются от равновесных. При этом в расчётах пограничного слоя на внешней границе вместо энтальпии газа задаются значения температуры и концентраций, полученные из расчёта уравнений Навье-Стокса;

- пограничный слой на моделях, помещенных в плазменную струю ВЧ плазмотрона остаётся неравновесным в широком диапазоне давлений и энерговкладов. Дополнительным параметром, позволяющим регулировать степень неравновесности, является размер моделей. Всё это позволяет использовать установки такого типа для широкого спектра экспериментальных исследований, в частности определения каталитической активности материалов и покрытий в отношении реакций рекомбинации атомов азота и кислорода в потоках азота и воздуха или атомов О и молекул СО в марсианской атмосфере;

- $\quad$ в разрядной камере картины течения и распределения параметров для атомарных и молекулярных газов сильно различаются. Это связано с различными механизмами образо- 
вания в области разряда «затравочных» электронов. Кроме этого, энергообмен электронов с молекулами в молекулярных газах в области разряда протекает с образованием отрицательного молекулярного иона $\mathrm{N}_{2}^{-}$, который быстро распадается на колебательновозбуждённую молекулу и электрон с меньшей энергией. Этот процесс препятствует значительному превышению температуры электронов над колебательной температурой молекул в области разряда.

Разработанный программный комплекс широко используется как при предварительном анализе возможностей моделирования условий теплообмена образцов ТЗМ, так и при интерпретации экспериментальных данных и определении наиболее важных характеристик материалов, таких как термостойкость и каталитичность.

\section{Литература}

1. Gordeev A.N., Kolesnikov A.F., Yakushin M.I. An Induction Plasma Application to «Buran» Heat Protection Tiles Ground Tests // SAMPLE Journal. 1992. V. 28, No. 3, pp. 29-33.

2. Залогин Г.Н., Землянский Б.А., Кнотько В.Б., Мурзинов И.Н., Румынский А.Н., Кузьмин Л.А. Высокочастотный плазмотрон - установка для исследований аэрофизических проблем с использованием высокоэнтальпийных газовых потоков // Космонавтика и ракетостроение. -1994. № 2.С.2232.

3. Жестков Б.Е., Исследование термохимической устойчивости теплозащитных материалов // Учёные записки ЦАГИ, 2014, т. XLV, №5.C.62-77.

4. Низкотемпературная плазма, том 6. ВЧ- и СВЧ-плазмотроны. Под ред. С.В. Дресвина и В.Д. Русанова. -Новосибирск: «Наука. Сибирское отделение». 1992, 317 с.

5. Boulos M.I., Cagne R., Barnes R.M. Effect of swirl and confinement of the flow and temperature field in an inductively coupled RF Plasma // Canadian J. Chem. Eng. 1980. V.58, No.3, pp.367-375.

6. Васильевский С.А., Колесников А.Ф. Численное моделирование равновесной индукционной плазмы в цилиндрическом канале плазмотрона // Изв. АН СССР. МЖГ. -2000. №5. С. 164-173.

7. Власов В.И. Теоретические исследования течения высокотемпературного газа в разрядной и рабочей камерах ВЧ-плазмотрона // Космонавтика и ракетостроение. - 2001. № 23. С.18-26.

8. Сёмин В.А. К теории неравновесного индукционного высокочастотного разряда в потоке газа // Изв. АН СССР. МЖГ. -1991. №2. С.153-160.

9. Boulos M.I. The inductively coupled radio-frequency plasma // Pure and Applied Chemistry. 1985. V.57, No. 9, pp.1321-1352.

10. Abele D.V., Degres G. Numerical of high-pressure air inductive plasmas under thermal and chemical nonequilibrium // AIAA Paper, No.2000-2416, 2000.

11. Власов В.И. Численное моделирование неравновесного течения в разрядной камере ВЧ-плазмотрона // Фундаментальные проблемы высокоскоростных течений. Тезисы докладов международной конф. ЦАГИ. 2004.

12. Lenzner S., Auweter-Kurtz M., Heiermann J., Sleziona P.C. Energy Partitions in Inductively Heated Plasma Sources for Reentry Simulations //Journal of Thermophysics and Heat Transfer, v.14, no.3, 2000, pp.388-395.

13. Sumi T., Fujita K., Ito T., Kurotaki T. Experimental evaluation of inductively coupled plasma flow analysis code // AIAA Paper 2005-175, 2005.

14. Tanaka Y. Two-temperature chemically non-equilibrium modeling of high-power $\mathrm{Ar}-\mathrm{N}_{2}$ inductively coupled plasmas at atmospheric pressure // Journal of Physics D: Applied Physics, v.37, 2004, pp.1190-1205.

15. Atsuchi N., Shigeta M., Watanabe T. Modeling of non-equilibrium argon-oxygen induction plasmas under atmospheric pressure // International Journal of Heat and Mass Transfer, v.49, 2006, pp.1073-1082.

16. Сахаров В.И. Численное моделирование термически и химически неравновесных течений и теплообмена в недорасширенных струях индукционного плазмотрона // Изв. РАН. МЖГ. 2007. № 6. C.157-168. 
17. Башкин В.А., Егоров И.В., Жестков Б.Е., Шведченко В.В. Численное исследование поля течения и теплообмена в тракте высокотемпературной аэродинамической установки // ТВТ, 2008, т.46, №5, С.771-783.

18. Василевский Э.Б., Жестков Б.Е., Сахаров В.И. Численное моделирование и эксперимент на индукционном плазмотроне АДТ ВАТ-104 // Ученые записки ЦАГИ, 2016, т. XLVII, №5, С.3-13.

19. Райзер Ю.П. Физика газового разряда. - 3-е изд. перераб. и доп., Долгопрудный: Издательский Дом «Интеллект», 2009. 736 с.

20. ЛандауЛ.Д., Лифшиц Е.М. Электродинамика сплошных сред. - М.: Наука. 1982, 620 с.

21. Власов В.И., Залогин Г.Н., Рудин Н.Ф. Экспериментальное определение энергетических параметров плазменного потока в установке с высокочастотным нагревом газа // Физико-химическая кинетика в газовой динамике. 2016. V.17 (2). http://chemphys.edu.ru/issues/2016-17-2/articles/651/

22. Землянский Б.А., Лунёв В.В., Власов В.И. и др. Конвективный теплообмен летательных аппаратов. Под редакцией Б.А. Землянского. - М.: ФИЗМАТЛИТ. 2014, 380 с.

23. Ковалев В.Л. Гетерогенные каталитические процессы в аэротермодинамике. - М.: ФИЗМАТЛИТ, 2002. $-224 \mathrm{c}$.

24. Брылкин Ю.В., Власов В.И., Залогин Г.Н., Кусов А.Л., Рудин Н.Ф.Экспериментальные исследования влияния структуры поверхности материалов на их каталитическую активность // Физико-химическая кинетика в газовой динамике. 2016. Том 17, вып. 1. hppt://www.chemphys. edu.ru/pdf/2016-17-01-004.pdf

25. Колесников А.Ф., Сахаров В.И. Корреляция условий теплообмена модели в недорасширенных струях диссоциированного углекислого газа и при гиперзвуковом обтекании сферы в марсианской атмосфере // Известия РАН. МЖГ, 2015, № 4.С. 131-138.

26. Колесников А.Ф., Сахаров В.И. Подобие теплообмена модели в недорасширенных струях диссоциированного воздуха в ВЧ-плазмотроне и при обтекании сферы высокоскоростным потоком в земной атмосфере // Известия РАН. МЖГ, 2016, № 3. С. 110-116.

27. Власов В.И., Залогин Г.Н., Ковалёв Р.В., Рудин Н.Ф. Физико-химическая модель смеси $\mathrm{CO}_{2}+\mathrm{N}_{2}$ при высоких температурах //Физико-химическая кинетика в газовой динамике. 2018. Т.19, вып.3. http://chemphys.edu.ru/issues/2018-19-3/articles/774/ 